\title{
WAVE AND KLEIN-GORDON EQUATIONS ON HYPERBOLIC SPACES
}

\author{
JEAN-PHILIPPE ANKER AND VITTORIA PIERFELICE
}

\begin{abstract}
We consider the Klein-Gordon equation associated with the LaplaceBeltrami operator $\Delta$ on real hyperbolic spaces of dimension $n \geq 2$; as $\Delta$ has a spectral gap, the wave equation is a particular case of our study. After a careful kernel analysis, we obtain dispersive and Strichartz estimates for a large family of admissible couples. As an application, we prove global well-posedness results for the corresponding semilinear equation with low regularity data.
\end{abstract}

\section{INTRODUCTION}

Dispersive properties of the wave and other evolution equations have been proved very useful in the study of nonlinear problems. The theory is well established for the Euclidean wave equation in dimension $n \geq 3$ :

$$
\left\{\begin{array}{l}
\partial_{t}^{2} u(t, x)-\Delta_{x} u(t, x)=F(t, x), \\
u(0, x)=f(x),\left.\partial_{t}\right|_{t=0} u(t, x)=g(x) .
\end{array}\right.
$$

The following Strichartz estimates hold for solutions $u$ to the Cauchy problem (1) :

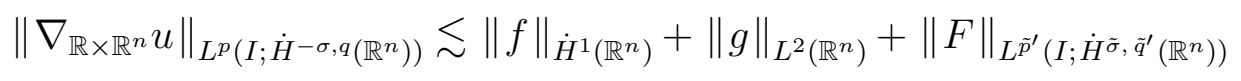

on any (possibly unbounded) time interval $I \subseteq \mathbb{R}$, under the assumptions that

$$
\sigma \geq \frac{n+1}{2}\left(\frac{1}{2}-\frac{1}{q}\right), \quad \tilde{\sigma} \geq \frac{n+1}{2}\left(\frac{1}{2}-\frac{1}{\tilde{q}}\right),
$$

and the couples $(p, q),(\tilde{p}, \tilde{q}) \in[2, \infty] \times[2, \infty)$ satisfy

$$
\frac{2}{p}+\frac{n-1}{q}=\frac{n-1}{2}, \quad \frac{2}{\tilde{p}}+\frac{n-1}{\tilde{q}}=\frac{n-1}{2} .
$$

We refer to [1] and 22] for more details.

These estimates serve as a tool for several existence results about the nonlinear wave equation in the Euclidean setting. The problem of finding minimal regularity conditions on the initial data ensuring local well-posedness for semilinear wave equations was addressed in [21] and then almost completely answered in [25, 22] (see Figure 5 in Section

Date: June 7, 2018.

2000 Mathematics Subject Classification. 35L05, 43A85 ; 22E30, 35L71, 43A90, 47J35, 58D25, 58J45, $81 \mathrm{Q} 05$.

Key words and phrases. Hyperbolic space, wave kernel, semilinear wave equation, semilinear KleinGordon equation, dispersive estimate, Strichartz estimate, global well-posedness. 
6). In general local solutions cannot be extended to global ones, unless further assumptions are made on the nonlinearity or on the initial data. A successful machinery was developed towards the global existence of small solutions to the semilinear wave equation

$$
\left\{\begin{array}{l}
\partial_{t}^{2} u(t, x)-\Delta_{x} u(t, x)=F(u), \\
u(0, x)=f(x),\left.\partial_{t}\right|_{t=0} u(t, x)=g(x),
\end{array}\right.
$$

with nonlinearities

$$
F(u) \sim|u|^{\gamma} \text { near } 0 .
$$

The results depend on the space dimension $n$. After the pioneer work [20] of John in dimension $n=3$, Strauss conjectured in [31] that the problem (3) is globally well posed in dimension $n \geq 2$ for small initial data provided

$$
\gamma>\gamma_{0}=\frac{1}{2}+\frac{1}{n-1}+\sqrt{\left(\frac{1}{2}+\frac{1}{n-1}\right)^{2}+\frac{2}{n-1}} .
$$

On one hand, the negative part of the conjecture was established by Sideris [30], who proved blow up for generic data (and nonlinearities satisfying $F(u) \gtrsim|u|^{\gamma}$ ) when $\gamma<\gamma_{0}$. On the other hand, the positive part of the conjecture was proved for any dimension in several steps (see e.g. [23], [10], [6], and [9] for a comprehensive survey).

Analogous results hold for the Klein-Gordon equation

$$
\partial_{t}^{2} u(t, x)-\Delta_{x} u(t, x)+u(t, x)=F(t, x),
$$

though its study has not been carried out as thoroughly as for the wave equation; in particular the sharpness of several well-posedness results is yet unknown (see [4], [12], [26], [28] and the references therein).

In view of the rich Euclidean theory, it is natural to look at the corresponding equations on more general manifolds. Here we consider real hyperbolic spaces $\mathbb{H}^{n}$, which are the most simple examples of noncompact Riemannian manifolds with negative curvature. For geometric reasons, we expect better dispersive properties hence stronger results than in the Euclidean setting.

Consider the wave equation associated to the Laplace-Beltrami operator $\Delta=\Delta_{\mathbb{H}^{n}}$ on $\mathbb{H}^{n}$ :

$$
\left\{\begin{array}{l}
\partial_{t}^{2} u(t, x)-\Delta_{x} u(t, x)=F(t, x), \\
u(0, x)=f(x),\left.\partial_{t}\right|_{t=0} u(t, x)=g(x),
\end{array}\right.
$$

The operator $-\Delta$ is positive on $L^{2}\left(\mathbb{H}^{n}\right)$ and its $L^{2}$-spectrum is the half-line $\left[\rho^{2},+\infty\right)$, where $\rho=\frac{n-1}{2}$. Thus (66) may be considered as a special case of the following family of Klein-Gordon equations

$$
\left\{\begin{array}{l}
\partial_{t}^{2} u(t, x)-\Delta_{x} u(t, x)+c u(t, x)=F(t, x), \\
u(0, x)=f(x),\left.\partial_{t}\right|_{t=0} u(t, x)=g(x),
\end{array}\right.
$$

where

$$
c \geq-\rho^{2}=-\frac{(n-1)^{2}}{4}
$$

is a constant. In the limit case $c=-\rho^{2}$, (7) is called the shifted wave equation. 
In [29] Pierfelice obtained Strichartz estimates for the non-shifted wave equation (6) with radial data on a class of Riemannian manifolds containing all hyperbolic spaces. The wave equation (6) was also investigated on the 3-dimensional hyperbolic space by Metcalfe and Taylor [27], who proved dispersive and Strichartz estimates with applications to small data global well-posedness for the semilinear wave equation. In his recent thesis [13], Hassani obtains a first set of results on noncompact Riemannian symmetric spaces of higher rank.

To our knowledge, the shifted wave equation (7) in the limit case $c=-\rho^{2}$ was first considered by Fontaine [7, 8] in low dimensions $n=3$ and $n=2$. In [32] Tataru obtained dispersive estimates for the operators $\frac{\sin \left(t \sqrt{\Delta+\rho^{2}}\right)}{\sqrt{\Delta+\rho^{2}}}$ and $\cos \left(t \sqrt{\Delta+\rho^{2}}\right)$ acting on inhomogeneous Sobolev spaces on $\mathbb{H}^{n}$ and then transferred them to $\mathbb{R}^{n}$ in order to get well-posedness results for the Euclidean semilinear wave equation (see also [9]). Complementary results were obtained by A. Ionescu [18, who investigated $L^{q} \rightarrow L^{q}$ Sobolev estimates for the above operators on all hyperbolic spaces.

A more detailed analysis of the shifted wave equation was carried out in [3]. There Strichartz estimates were obtained for a wider range of couples than in the Euclidean setting and consequently stronger well-posedness results were shown to hold for the nonlinear equations. Corresponding results for the Schrödinger equation were obtained in [1], 2] and [19].

In the present paper we study the family of equations (7) in the remaining range $c>-\rho^{2}$ and in dimension $n \geq 2$, which includes the particular case $c=0$ and $n=3$ considered in [27]. In order to state and describe our results, it is convenient to rewrite the constant (8) as follows:

$$
c=\kappa^{2}-\rho^{2} \quad \text { with } \quad \kappa>0,
$$

and to introduce the operator

$$
D=\sqrt{-\Delta-\rho^{2}+\kappa^{2}}
$$

as well as

$$
\widetilde{D}=\sqrt{-\Delta-\rho^{2}+\tilde{\kappa}^{2}}
$$

where $\tilde{\kappa}>\rho$ is another fixed constant. Thus our family of equations (77) becomes

$$
\left\{\begin{array}{l}
\partial_{t}^{2} u(t, x)-D_{x}^{2} u(t, x)=F(t, x), \\
u(0, x)=f(x),\left.\partial_{t}\right|_{t=0} u(t, x)=g(x),
\end{array}\right.
$$

the wave equation (6) corresponding to the choice $\kappa=\rho$ and the shifted wave equation to the limit case $\kappa=0$.

Let us now describe the content of this paper and present our main results, that we state for simplicity in dimension $n \geq 3$. In Section 2, we recall the basis tools of spherical Fourier analysis on real hyperbolic spaces $\mathbb{H}^{n}$. After analyzing carefully the integral kernel of the half wave operator

$$
W_{t}^{\sigma}=\widetilde{D}^{-\sigma} e^{i t D}
$$


in Section 3, we prove in Section 4 the following dispersive estimates, which combine the small time estimates [3] for the shifted wave equation and the large time estimates [1] for the Schrödinger equation :

$$
\left\|W_{t}^{\sigma}\right\|_{L^{q} \rightarrow L^{q^{\prime}}} \lesssim \begin{cases}|t|^{-(n-1)\left(\frac{1}{2}-\frac{1}{q}\right)} & \text { if } 0<|t|<1, \\ |t|^{-\frac{3}{2}} & \text { if }|t| \geq 1,\end{cases}
$$

where $2<q<\infty$ and $\sigma \geq(n+1)\left(\frac{1}{2}-\frac{1}{q}\right)$. Notice that we don't deal with the limit case $q=\infty$, where Metcalfe and Taylor [27] have obtained an $H^{1} \rightarrow B M O$ estimate in dimension $n=3$.

In Section 5 we deduce the Strichartz estimates

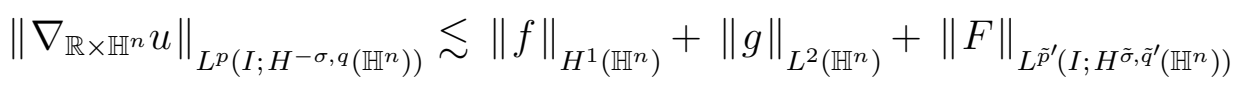

for solutions $u$ to (12). Here $I \subseteq \mathbb{R}$ is any time interval,

$$
\sigma \geq \frac{n+1}{2}\left(\frac{1}{2}-\frac{1}{q}\right), \quad \tilde{\sigma} \geq \frac{n+1}{2}\left(\frac{1}{2}-\frac{1}{\tilde{q}}\right),
$$

and the couples $\left(\frac{1}{p}, \frac{1}{q}\right),\left(\frac{1}{\tilde{p}}, \frac{1}{\tilde{q}}\right)$ belong to the triangle

$$
\left\{\left(\frac{1}{p}, \frac{1}{q}\right) \in\left(0, \frac{1}{2}\right] \times\left(0, \frac{1}{2}\right) \mid \frac{1}{p} \geq \frac{n-1}{2}\left(\frac{1}{2}-\frac{1}{q}\right)\right\} \cup\left\{\left(0, \frac{1}{2}\right)\right\} .
$$

These estimates are similar to those obtained in [3] for the shifted wave equation, except that they involve standard Sobolev spaces and no exotic ones. Notice that the range (13) of admissible couples for $\mathbb{H}^{n}$ is substantially wider than the range (2) for $\mathbb{R}^{n}$, which corresponds to the lower edge of the triangle (13).

In Section 6 we apply the results of the previous sections to the problem of global existence with small data for the corresponding semilinear equations. In contrast with the Euclidean case, where the range of admissible nonlinearities $F(u) \sim|u|^{\gamma}$ is restricted to $\gamma>\gamma_{0}$, we prove global well-posedness for powers $\gamma>1$ arbitrarily close to 1 . This result improves in particular [27], where global well-posedness for (6) was obtained in the case $n=3$ and $\kappa=\rho$ under the assumption $\gamma>5 / 3$.

In conclusion, the fact that better results hold for $\mathbb{H}^{n}$ than for $\mathbb{R}^{n}$ may be regarded as a consequence of the stronger dispersion properties of waves in negative curvature. Besides our results extend to the more general setting of Damek-Ricci spaces, as done in [2] for the Schrödinger equation and in [3] for the shifted wave equation.

\section{SPHERICAL ANALYSIS ON REAL HYPERBOLIC SPACES}

In this paper, we consider the simplest class of Riemannian symmetric spaces of the noncompact type, namely real hyperbolic spaces $\mathbb{H}^{n}$ of dimension $n \geq 2$. We refer to Helgason's books [14, 15, 16] and to Koornwinder's survey [24] for their algebraic structure and geometric properties, as well as for harmonic analysis on these spaces, and we shall be content with the following information. $\mathbb{H}^{n}$ can be realized as the symmetric space $G / K$, where $G=\mathrm{SO}(1, n)_{0}$ and $K=\mathrm{SO}(n)$. In geodesic polar coordinates, the Laplace-Beltrami operator on $\mathbb{H}^{n}$ writes

$$
\Delta_{\mathbb{H}^{n}}=\partial_{r}^{2}+(n-1) \operatorname{coth} r \partial_{r}+\sinh ^{-2} r \Delta_{\mathbb{S}^{n-1}} .
$$


The spherical functions $\varphi_{\lambda}$ on $\mathbb{H}^{n}$ are normalized radial eigenfunctions of $\Delta=\Delta_{\mathbb{H}^{n}}$ :

$$
\left\{\begin{array}{l}
\Delta \varphi_{\lambda}=-\left(\lambda^{2}+\rho^{2}\right) \varphi_{\lambda} \\
\varphi_{\lambda}(0)=1
\end{array}\right.
$$

where $\lambda \in \mathbb{C}$ and $\rho=\frac{n-1}{2}$. They can be expressed in terms of special functions:

$$
\varphi_{\lambda}(r)=\phi_{\lambda}^{\left(\frac{n}{2}-1,-\frac{1}{2}\right)}(r)={ }_{2} F_{1}\left(\frac{\rho}{2}+i \frac{\lambda}{2}, \frac{\rho}{2}-i \frac{\lambda}{2} ; \frac{n}{2} ;-\sinh ^{2} r\right)
$$

where $\phi_{\lambda}^{(\alpha, \beta)}$ denotes the Jacobi functions and ${ }_{2} F_{1}$ the Gauss hypergeometric function. In the sequel we shall use the Harish-Chandra formula

$$
\varphi_{\lambda}(r)=\int_{K} d k e^{-(\rho+i \lambda) \mathrm{H}\left(a_{-r} k\right)}
$$

and the basic estimate

$$
\left|\varphi_{\lambda}(r)\right| \leq \varphi_{0}(r) \lesssim(1+r) e^{-\rho r} \quad \forall \lambda \in \mathbb{R}, r \geq 0 .
$$

We shall also use the Harish-Chandra expansion

$$
\varphi_{\lambda}(r)=\mathbf{c}(\lambda) \Phi_{\lambda}(r)+\mathbf{c}(-\lambda) \Phi_{-\lambda}(r) \quad \forall \lambda \in \mathbb{C} \backslash \mathbb{Z}, r>0,
$$

where the Harish-Chandra $\mathbf{c}-$ function is given by

$$
\mathbf{c}(\lambda)=\frac{\Gamma(2 \rho)}{\Gamma(\rho)} \frac{\Gamma(i \lambda)}{\Gamma(i \lambda+\rho)}
$$

and

$$
\begin{aligned}
\Phi_{\lambda}(r) & =(2 \sinh r)^{i \lambda-\rho}{ }_{2} F_{1}\left(\frac{\rho}{2}-i \frac{\lambda}{2},-\frac{\rho-1}{2}-i \frac{\lambda}{2} ; 1-i \lambda ;-\sinh ^{-2} r\right) \\
& =(2 \sinh r)^{-\rho} e^{i \lambda r} \sum_{k=0}^{+\infty} \Gamma_{k}(\lambda) e^{-2 k r} \\
& \sim e^{(i \lambda-\rho) r} \quad \text { as } r \rightarrow+\infty .
\end{aligned}
$$

The coefficients $\Gamma_{k}(\lambda)$ in the expansion (18) are rational functions of $\lambda \in \mathbb{C}$, which satisfy the recurrence formula

$$
\left\{\begin{array}{l}
\Gamma_{0}(\lambda)=1, \\
\Gamma_{k}(\lambda)=\frac{\rho(\rho-1)}{k(k-i \lambda)} \sum_{j=0}^{k-1}(k-j) \Gamma_{j}(\lambda) .
\end{array}\right.
$$

Their classical estimates were improved as follows in [2, Lemma 2.1].

Lemma 2.1. Let $0<\varepsilon<1$ and $\Omega_{\varepsilon}=\{\lambda \in \mathbb{C}|\operatorname{Re} \lambda \leq \varepsilon| \lambda \mid, \operatorname{Im} \lambda \leq-1+\varepsilon\}$. Then there exist $\nu \geq 0$ and, for every $\ell \in \mathbb{N}, C_{\ell} \geq 0$ such that

$$
\left|\partial_{\lambda}^{\ell} \Gamma_{k}(\lambda)\right| \leq C_{\ell} k^{\nu}(1+|\lambda|)^{-\ell-1} \quad \forall k \in \mathbb{N}^{*}, \lambda \in \mathbb{C} \backslash \Omega_{\varepsilon} .
$$

Under suitable assumptions, the spherical Fourier transform of a bi- $K$-invariant function $f$ on $G$ is defined by

$$
\mathcal{H} f(\lambda)=\int_{G} d g f(g) \varphi_{\lambda}(g)
$$


and the following inversion formula holds :

$$
f(x)=\text { const. } \int_{0}^{+\infty} d \lambda|\mathbf{c}(\lambda)|^{-2} \mathcal{H} f(\lambda) \varphi_{\lambda}(x) .
$$

Here is a well-known estimate of the Plancherel density:

$$
|\mathbf{c}(\lambda)|^{-2} \lesssim|\lambda|^{2}(1+|\lambda|)^{n-3} \quad \forall \lambda \in \mathbb{R} .
$$

Via the spherical Fourier transform, the Laplace-Beltrami operator $\Delta$ corresponds to

$$
-\lambda^{2}-\rho^{2},
$$

hence the operators $D=\sqrt{-\Delta-\rho^{2}+\kappa^{2}}$ and $\widetilde{D}=\sqrt{-\Delta-\rho^{2}+\tilde{\kappa}^{2}}$ to

$$
\sqrt{\lambda^{2}+\kappa^{2}} \text { and } \sqrt{\lambda^{2}+\tilde{\kappa}^{2}} \text {. }
$$

\section{KeRnel EStimates}

In this section we derive pointwise estimates for the radial convolution kernel $w_{t}^{\sigma}$ of the operator $W_{t}^{\sigma}=\widetilde{D}^{-\sigma} e^{i t D}$, for suitable exponents $\sigma \in \mathbb{R}$. By the inversion formula of the spherical Fourier transform,

$$
w_{t}^{\sigma}(r)=\text { const. } \int_{-\infty}^{+\infty} d \lambda|\mathbf{c}(\lambda)|^{-2}\left(\lambda^{2}+\tilde{\kappa}^{2}\right)^{-\frac{\sigma}{2}} \varphi_{\lambda}(r) e^{i t \sqrt{\lambda^{2}+\kappa^{2}}} .
$$

Contrarily to the Euclidean case, this kernel has different behaviors, depending whether $t$ is small or large, and therefore we cannot use any rescaling. Let us split up

$$
\begin{aligned}
w_{t}^{\sigma}(r) & =w_{t}^{\sigma, 0}(r)+w_{t}^{\sigma, \infty}(r) \\
& =\text { const. } \int_{-\infty}^{+\infty} d \lambda \chi_{0}(\lambda)|\mathbf{c}(\lambda)|^{-2}\left(\lambda^{2}+\tilde{\kappa}^{2}\right)^{-\frac{\sigma}{2}} \varphi_{\lambda}(r) e^{i t \sqrt{\lambda^{2}+\kappa^{2}}} \\
& + \text { const. } \int_{-\infty}^{+\infty} d \lambda \chi_{\infty}(\lambda)|\mathbf{c}(\lambda)|^{-2}\left(\lambda^{2}+\tilde{\kappa}^{2}\right)^{-\frac{\sigma}{2}} \varphi_{\lambda}(r) e^{i t \sqrt{\lambda^{2}+\kappa^{2}}}
\end{aligned}
$$

using smooth even cut-off functions $\chi_{0}$ and $\chi_{\infty}$ on $\mathbb{R}$ such that

$$
\chi_{0}(\lambda)+\chi_{\infty}(\lambda)=1 \quad \text { and } \quad \begin{cases}\chi_{0}(\lambda)=1 & \forall|\lambda| \leq \kappa \\ \chi_{\infty}(\lambda)=1 & \forall|\lambda| \geq \kappa+1 .\end{cases}
$$

We shall first estimate $w_{t}^{\sigma, 0}$ and next a variant of $w_{t}^{\sigma, \infty}$. The kernel $w_{t}^{\sigma, \infty}$ has indeed a logarithmic singularity on the sphere $r=t$ when $\sigma=\frac{n+1}{2}$. We bypass this problem by considering the analytic family of operators

$$
\widetilde{W}_{t}^{\sigma, \infty}=\frac{e^{\sigma^{2}}}{\Gamma\left(\frac{n+1}{2}-\sigma\right)} \chi_{\infty}(D) \widetilde{D}^{-\sigma} e^{i t D}
$$

in the vertical strip $0 \leq \operatorname{Re} \sigma \leq \frac{n+1}{2}$ and the corresponding kernels

$$
\widetilde{w}_{t}^{\sigma, \infty}(r)=\text { const. } \frac{e^{\sigma^{2}}}{\Gamma\left(\frac{n+1}{2}-\sigma\right)} \int_{-\infty}^{+\infty} d \lambda \chi_{\infty}(\lambda)|\mathbf{c}(\lambda)|^{-2}\left(\lambda^{2}+\tilde{\kappa}^{2}\right)^{-\frac{\sigma}{2}} \varphi_{\lambda}(r) e^{i t \sqrt{\lambda^{2}+\kappa^{2}}} .
$$


Notice that the Gamma function (which occurs naturally in the theory of Riesz distributions) will allow us to deal with the boundary point $\sigma=\frac{n+1}{2}$, while the exponential function yields boundedness at infinity in the vertical strip.

\subsection{Estimate of $w_{t}^{0}=w_{t}^{\sigma, 0}$.}

Theorem 3.1. Let $\sigma \in \mathbb{R}$. The following pointwise estimates hold for the kernel $w_{t}^{0}$ :

(i) For every $t \in \mathbb{R}$ and $r \geq 0$, we have

$$
\left|w_{t}^{0}(r)\right| \lesssim \varphi_{0}(r)
$$

(ii) Assume that $|t| \geq 2$. Then, for every $0 \leq r \leq \frac{|t|}{2}$, we have

$$
\left|w_{t}^{0}(r)\right| \lesssim|t|^{-\frac{3}{2}}(1+r) \varphi_{0}(r)
$$

Proof. Recall that

$$
w_{t}^{0}(r)=\text { const. } \int_{-\kappa-1}^{\kappa+1} d \lambda \chi_{0}(\lambda)|\mathbf{c}(\lambda)|^{-2}\left(\lambda^{2}+\tilde{\kappa}^{2}\right)^{-\frac{\sigma}{2}} \varphi_{\lambda}(r) e^{i t \sqrt{\lambda^{2}+\kappa^{2}}} .
$$

By symmetry we may assume that $t>0$.

(i) It follows from the estimates (15) and (20) that

$$
\left|w_{t}^{0}(r)\right| \lesssim \int_{-\kappa-1}^{\kappa+1} d \lambda \lambda^{2} \varphi_{0}(r) \lesssim \varphi_{0}(r)
$$

We prove (ii) by substituting in (22) the first integral representation of $\varphi_{\lambda}$ in (14) and by reducing in this way to Fourier analysis on $\mathbb{R}$. Specifically,

$$
w_{t}^{0}(r)=\int_{K} d k e^{-\rho \mathrm{H}\left(a_{-r} k\right)} \int_{-\infty}^{+\infty} d \lambda a(\lambda) e^{i t\left(\sqrt{\lambda^{2}+\kappa^{2}}-\frac{\mathrm{H}\left(a_{-r} k\right) \lambda}{t}\right)},
$$

where $a(\lambda)=$ const. $\chi_{0}(\lambda)|\mathbf{c}(\lambda)|^{-2}\left(\lambda^{2}+\tilde{\kappa}^{2}\right)^{-\frac{\sigma}{2}}$. Since

$$
\int_{K} d k e^{-\rho \mathrm{H}\left(a_{-r} k\right)}=\varphi_{0}(r)
$$

and $\left|\mathrm{H}\left(a_{-r} k\right)\right| \leq r$, it remains for us to estimate the oscillatory integral

$$
I(t, x)=\int_{-\infty}^{+\infty} d \lambda a(\lambda) e^{i t\left(\sqrt{\lambda^{2}+\kappa^{2}}-\frac{x \lambda}{t}\right)}
$$

by $|t|^{-\frac{3}{2}}(1+|x|)$. This is obtained by the method of stationary phase. More precisely, we apply Lemma A.1 in Appendix A, whose assumption (80) is fulfilled, according to (20). 


\subsection{Estimate of $\widetilde{w}_{t}^{\infty}=\widetilde{w}_{t}^{\sigma, \infty}$.}

Theorem 3.2. The following pointwise estimates hold for the kernel $\widetilde{w}_{t}^{\infty}$, uniformy in $\sigma \in \mathbb{C}$ with $\operatorname{Re} \sigma=\frac{n+1}{2}$ :

(i) Assume that $|t| \geq 2$. Then, for every $r \geq 0$, we have

$$
\left|\widetilde{w}_{t}^{\infty}(r)\right| \lesssim|t|^{-\infty}
$$

(ii) Assume that $0<|t| \leq 2$.

(a) If $r \geq 3$, then $\widetilde{w}_{t}^{\infty}(r)=\mathrm{O}\left(r^{-1} e^{-\rho r}\right)$.

(b) If $0 \leq r \leq 3$, then $\left|\widetilde{w}_{t}^{\infty}(r)\right| \lesssim \begin{cases}|t|^{-\frac{n-1}{2}} & \text { if } n \geq 3 \text {, } \\ |t|^{-\frac{1}{2}}(1-\log |t|) & \text { if } n=2 .\end{cases}$

By symmetry we may assume again $t>0$ throughout the proof of Theorem 3.2 . Proof of Theorem 3.2. $i$. By evenness we have

$$
\widetilde{w}_{t}^{\infty}(r)=2 \text { const. } \frac{e^{\sigma^{2}}}{\Gamma\left(\frac{n+1}{2}-\sigma\right)} \int_{0}^{+\infty} d \lambda \chi_{\infty}(\lambda)|\mathbf{c}(\lambda)|^{-2}\left(\lambda^{2}+\tilde{\kappa}^{2}\right)^{-\frac{\sigma}{2}} \varphi_{\lambda}(r) e^{i t \sqrt{\lambda^{2}+\kappa^{2}}} .
$$

If $0 \leq r \leq \frac{t}{2}$, we resume the proof of Theorem 3.1,ii, using Lemma A.2 instead of Lemma A.1, and conclude that

$$
\left|\widetilde{w}_{t}^{\infty}(r)\right| \lesssim t^{-\infty} \varphi_{0}(r)
$$

If $r \geq \frac{t}{2}$, we substitute in (23) the Harish-Chandra expansion (16) of $\varphi_{\lambda}(r)$ and reduce this way again to Fourier analysis on $\mathbb{R}$. Specifically, our task consists in estimating the expansion

$$
\widetilde{w}_{t}^{\infty}(r)=(\sinh r)^{-\rho} \sum_{k=0}^{+\infty} e^{-2 k r}\left\{I_{k}^{+, \infty}(t, r)+I_{k}^{-, \infty}(t, r)\right\}
$$

involving oscillatory integrals

$$
I_{k}^{ \pm, \infty}(t, r)=\int_{0}^{+\infty} d \lambda a_{k}^{ \pm}(\lambda) e^{i\left(t \sqrt{\lambda^{2}+\kappa^{2}} \pm r \lambda\right)}
$$

with amplitudes

$$
a_{k}^{ \pm}(\lambda)=2 \text { const. } \frac{e^{\sigma^{2}}}{\Gamma\left(\frac{n+1}{2}-\sigma\right)} \chi_{\infty}(\lambda) \mathbf{c}(\mp \lambda)^{-1}\left(\lambda^{2}+\tilde{\kappa}^{2}\right)^{-\frac{\sigma}{2}} \Gamma_{k}( \pm \lambda) .
$$

Notice that $a_{k}^{ \pm}(\lambda)$ is a symbol of order

$$
d= \begin{cases}-1 & \text { if } k=0 \\ -2 & \text { if } k \in \mathbb{N}^{*}\end{cases}
$$

uniformly in $\sigma \in \mathbb{C}$ with $\operatorname{Re} \sigma=\frac{n+1}{2}$. This follows indeed from the expression (17) of the c-function and from the estimate (19) of the coefficients $\Gamma_{k}$. Consequently the integrals

$$
I_{k}^{ \pm, \infty}(t, r)=\mathrm{O}\left(k^{\nu}\right)
$$


are easy to estimate when $k>0$, while $I_{0}^{+, \infty}(t, r)$ and especially $I_{0}^{-, \infty}(t, r)$ require more work. As far as it is concerned, we integrate by parts

$$
I_{0}^{+, \infty}(t, r)=\int_{0}^{+\infty} d \lambda a_{0}^{+}(\lambda) e^{i t \phi(\lambda)},
$$

using $e^{i t \phi(\lambda)}=\frac{1}{i t \phi^{\prime}(\lambda)} \frac{\partial}{\partial \lambda} e^{i t \phi(\lambda)}$ and the following properties of $\phi(\lambda)=\sqrt{\lambda^{2}+\kappa^{2}}+\frac{r}{t} \lambda$ :

- $\phi^{\prime}(\lambda)=\frac{\lambda}{\sqrt{\lambda^{2}+\kappa^{2}}}+\frac{r}{t} \geq \frac{r}{t} \geq \frac{1}{2}$

- $\phi^{\prime \prime}(\lambda)=\kappa^{2}\left(\lambda^{2}+\kappa^{2}\right)^{-\frac{3}{2}}$ is a symbol of order -3 .

We obtain this way

$$
I_{0}^{+, \infty}(t, r)=\mathrm{O}\left(r^{-1}\right)
$$

and actually

$$
I_{0}^{+, \infty}(t, r)=\mathrm{O}\left(r^{-\infty}\right)
$$

by repeated integrations by parts. Let us turn to the last integral, that we rewrite as follows :

$$
I_{0}^{-, \infty}(t, r)=\int_{0}^{+\infty} d \lambda a_{0}^{-}(\lambda) e^{i t\left(\sqrt{\lambda^{2}+\kappa^{2}}-\lambda\right)} e^{i(t-r) \lambda} .
$$

After performing an integration by parts based on $e^{i(t-r) \lambda}=\frac{1}{i(t-r)} \frac{\partial}{\partial \lambda} e^{i(t-r) \lambda}$ and by using the fact that

$$
\psi(\lambda)=\sqrt{\lambda^{2}+\kappa^{2}}-\lambda=\frac{\kappa^{2}}{\sqrt{\lambda^{2}+\kappa^{2}}+\lambda}
$$

is a symbol of order -1 , we obtain

$$
I_{0}^{-, \infty}(t, r)=\mathrm{O}\left(\frac{t}{|r-t|}\right) .
$$

This estimate is enough for our purpose, as long as $r$ stays away from $t$. If $|r-t| \leq 1$, let us split up

$$
e^{i t \psi(\lambda)}=1+\mathrm{O}(t \psi(\lambda))
$$

and

$$
I_{0}^{-, \infty}(t, r)=\int_{0}^{+\infty} d \lambda a_{0}^{-}(\lambda) e^{i(t-r) \lambda}+\mathrm{O}(t)
$$

accordingly. The remaining integral was estimated in [2], more precisely at the end of the proof Theorem 4.2.ii :

$$
\int_{0}^{+\infty} d \lambda a_{0}^{-}(\lambda) e^{i(t-r) \lambda}=\mathrm{O}(1)
$$

By combining the estimates (26), (27), (29), (30), (31), we deduce from (25) that

$$
\left|\widetilde{w}_{t}^{\infty}(r)\right| \lesssim e^{-\rho r} t \lesssim t^{-\infty} \quad \forall r \geq \frac{t}{2} \geq 1
$$

uniformly in $\sigma \in \mathbb{C}$ with $\operatorname{Re} \sigma=\frac{n+1}{2}$. This concludes the proof of Theorem 3.2,i.

Let us turn to the small time estimates in Theorem 3.2 
Proof of Theorem 3.2. ii.a. Since $0<t \leq 2$ and $r \geq 3$, we can resume the proof of Theorem 3.2. i in the case $r \geq t+1 \geq \frac{t}{2}$. By using the expansion (25) and the estimates (26) , (27), (29), we obtain

$$
\left|\widetilde{w}_{t}^{\infty}(r)\right| \lesssim r^{-1} e^{-\rho r},
$$

uniformly in $\sigma \in \mathbb{C}$ with $\operatorname{Re} \sigma=\frac{n+1}{2}$. This concludes the proof of Theorem 3.2,ii.a.

Proof of Theorem 3.2 ii.b. Let us rewrite and expand (23) as follows:

$$
\begin{aligned}
\widetilde{w}_{t}^{\infty}(r) & =2 \text { const. } \frac{e^{\sigma^{2}}}{\Gamma\left(\frac{n+1}{2}-\sigma\right)} \int_{0}^{+\infty} d \lambda \chi_{\infty}(\lambda)|\mathbf{c}(\lambda)|^{-2}\left(\lambda^{2}+\tilde{\kappa}^{2}\right)^{-\frac{\sigma}{2}} e^{i t \psi(\lambda)} e^{i t \lambda} \varphi_{\lambda}(r) \\
& =\int_{0}^{+\infty} d \lambda a(\lambda) e^{i t \lambda} \varphi_{\lambda}(r)+\int_{0}^{+\infty} d \lambda b(\lambda) e^{i t \lambda} \varphi_{\lambda}(r),
\end{aligned}
$$

where $\psi$ is given by (28),

$$
a(\lambda)=2 \text { const. } \frac{e^{\sigma^{2}}}{\Gamma\left(\frac{n+1}{2}-\sigma\right)} \chi_{\infty}(\lambda)|\mathbf{c}(\lambda)|^{-2}\left(\lambda^{2}+\tilde{\kappa}^{2}\right)^{-\frac{\sigma}{2}}
$$

is a symbol of order $\frac{n-3}{2}$, uniformly in $\sigma \in \mathbb{C}$ with $\operatorname{Re} \sigma=\frac{n+1}{2}$, and

$$
b(\lambda)=2 \text { const. } \frac{e^{\sigma^{2}}}{\Gamma\left(\frac{n+1}{2}-\sigma\right)} \chi_{\infty}(\lambda)|\mathbf{c}(\lambda)|^{-2}\left(\lambda^{2}+\tilde{\kappa}^{2}\right)^{-\frac{\sigma}{2}}\left\{e^{i t \psi(\lambda)}-1\right\}
$$

is a symbol of $\frac{n-5}{2}$, uniformly in $0<t \leq 2$ and $\sigma \in \mathbb{C}$ with $\operatorname{Re} \sigma=\frac{n+1}{2}$. The first integral in (33) was analyzed in [2, Appendix C] and estimated there by

$$
C \begin{cases}t^{-\frac{n-1}{2}} & \text { if } n \geq 3, \\ t^{-\frac{1}{2}}(1-\log |t|) & \text { if } n=2 .\end{cases}
$$

The second integral is easier to estimate, for instance by $C t^{-\frac{n-2}{2}}$. This concludes the proof of Theorem 3.2,ii.b.

Remark 3.3. As far as local estimates of wave kernels are concerned, we might have used the Hadamard parametrix [17, § 17.4] instead of spherical analysis.

Remark 3.4. The kernel analysis carried out in this section still holds for the operators $D^{-\sigma} \widetilde{D}^{-\tilde{\sigma}} e^{i t D}$, provided we assume $\operatorname{Re} \sigma+\operatorname{Re} \widetilde{\sigma}=\frac{n+1}{2}$ in Theorem 3.2.

\section{Dispersive estimates}

In this section we obtain $L^{q^{\prime}} \rightarrow L^{q}$ estimates for the operator $W_{t}^{\sigma}=\widetilde{D}^{-\sigma} e^{i t D}$, which will be crucial role for our Strichartz estimates in next section. Let us split up its kernel $w_{t}^{\sigma}=w_{t}^{\sigma, 0}+w_{t}^{\sigma, \infty}$ as before. We will handle the contribution of $w_{t}^{\sigma, 0}$, using the pointwise estimates obtained in Subsection 3.1 and the following criterion (see for instance [2, Theorem 3.4]) based on the Kunze-Stein phenomenon.

Lemma 4.1. There exists a constant $C>0$ such that, for every radial measurable function $\kappa$ on $\mathbb{H}^{n}$, for every $2 \leq q<\infty$ and $f \in L^{q^{\prime}}\left(\mathbb{H}^{n}\right)$,

$$
\|f * \kappa\|_{L^{q}} \leq C_{q}\|f\|_{L^{q^{\prime}}}\left\{\int_{0}^{+\infty} d r(\sinh r)^{n-1}|\kappa(r)|^{\frac{q}{2}} \varphi_{0}(r)\right\}^{\frac{2}{q}} .
$$


For the second part $w_{t}^{\sigma, \infty}$, we resume the Euclidean approach, which consists in interpolating analytically between $L^{2} \rightarrow L^{2}$ and $L^{1} \rightarrow L^{\infty}$ estimates for the family of operators

$$
\widetilde{W}_{t}^{\sigma, \infty}=\frac{e^{\sigma^{2}}}{\Gamma\left(\frac{n+1}{2}-\sigma\right)} \chi_{\infty}(D) \widetilde{D}^{-\sigma} e^{i t D}
$$

in the vertical strip $0 \leq \operatorname{Re} \sigma \leq \frac{n+1}{2}$.

\subsection{Small time dispersive estimates.}

Theorem 4.2. Assume that $0<|t| \leq 2,2<q<\infty$ and $\sigma \geq(n+1)\left(\frac{1}{2}-\frac{1}{q}\right)$. Then,

$$
\left\|\widetilde{D}^{-\sigma} e^{i t D}\right\|_{L^{q^{\prime} \rightarrow L^{q}}} \lesssim \begin{cases}|t|^{-(n-1)\left(\frac{1}{2}-\frac{1}{q}\right)} & \text { if } n \geq 3, \\ |t|^{-\left(\frac{1}{2}-\frac{1}{q}\right)}(1-\log |t|)^{1-\frac{2}{q}} & \text { if } n=2 .\end{cases}
$$

Proof. We divide the proof into two parts, corresponding to the kernel decomposition $w_{t}=w_{t}^{0}+w_{t}^{\infty}$. By applying Lemma 4.1 and using the pointwise estimates in Theorem 3.1.i, we obtain on one hand

$$
\begin{aligned}
\left\|f * w_{t}^{0}\right\|_{L^{q}} & \lesssim\left\{\int_{0}^{+\infty} d r(\sinh r)^{n-1} \varphi_{0}(r)\left|w_{t}^{0}(r)\right|^{\frac{q}{2}}\right\}^{\frac{2}{q}}\|f\|_{L^{q^{\prime}}} \\
& \lesssim\left\{\int_{0}^{+\infty} d r(1+r)^{\frac{q}{2}+1} e^{-\left(\frac{q}{2}-1\right) \rho r}\right\}^{\frac{2}{q}}\|f\|_{L^{q^{\prime}}} \\
& \lesssim\|f\|_{L^{q^{\prime}}} \quad \forall f \in L^{q^{\prime}} .
\end{aligned}
$$

On the other hand, in order to estimate the $L^{q^{\prime}} \rightarrow L^{q}$ norm of $f \mapsto f * w_{t}^{\infty}$, we proceed by interpolation for the analytic family (34). If $\operatorname{Re} \sigma=0$, then

$$
\left\|f * \widetilde{w}_{t}^{\infty}\right\|_{L^{2}} \lesssim\|f\|_{L^{2}} \quad \forall f \in L^{2} .
$$

If $\operatorname{Re} \sigma=\frac{n+1}{2}$, we deduce from the pointwise estimates in Theorem 3.2. ii that

$$
\left\|f * \widetilde{w}_{t}^{\infty}\right\|_{L^{\infty}} \lesssim|t|^{-\frac{n-1}{2}}\|f\|_{L^{1}} \quad \forall f \in L^{1} .
$$

By interpolation we conclude for $\sigma=(n+1)\left(\frac{1}{2}-\frac{1}{q}\right)$ that

$$
\left\|f * w_{t}^{\infty}\right\|_{L^{q}} \lesssim|t|^{-(n-1)\left(\frac{1}{2}-\frac{1}{q}\right)}\|f\|_{L^{q^{\prime}}} \quad \forall f \in L^{q^{\prime}} .
$$

\subsection{Large time dispersive estimate.}

Theorem 4.3. Assume that $|t| \geq 2,2<q<\infty$ and $\sigma \geq(n+1)\left(\frac{1}{2}-\frac{1}{q}\right)$. Then

$$
\left\|\widetilde{D}^{-\sigma} e^{i t D}\right\|_{L^{q^{\prime} \rightarrow L^{q}}} \lesssim|t|^{-\frac{3}{2}}
$$

Proof. We divide the proof into three parts, corresponding to the kernel decomposition

$$
w_{t}=\mathbb{I}_{B\left(0, \frac{|t|}{2}\right)} w_{t}^{0}+\mathbb{I}_{\mathbb{H}^{n} \backslash B\left(0, \frac{|t|}{2}\right)} w_{t}^{0}+w_{t}^{\infty} .
$$


Estimate 1: By applying Lemma 4.1 and using the pointwise estimate in Theorem 3.1,ii, we obtain

$$
\begin{aligned}
\left\|f *\left\{\mathbb{I}_{B\left(0, \frac{|t|}{2}\right)} w_{t}^{0}\right\}\right\|_{L^{q}} & \lesssim\left\{\int_{0}^{\frac{|t|}{2}} d r(\sinh r)^{n-1} \varphi_{0}(r)\left|w_{t}^{0}(r)\right|^{\frac{q}{2}}\right\}^{\frac{2}{q}}\|f\|_{L^{q^{\prime}}} \\
& \lesssim \underbrace{\left\{\int_{0}^{\frac{|t|}{2}} d r(1+r)^{q+1} e^{-\left(\frac{q}{2}-1\right) \rho r}\right\}^{\frac{2}{q}}|t|^{-\frac{3}{2}}\|f\|_{L^{q^{\prime}}} \quad \forall f \in L^{q^{\prime}} .}_{<+\infty}
\end{aligned}
$$

Estimate 2: By applying Lemma 4.1 and using the pointwise estimate in Theorem 3.1.i, we obtain

$$
\begin{aligned}
\left\|f *\left\{\mathbb{I}_{\mathbb{H}^{n} \backslash B\left(0, \frac{|t|}{2}\right)} w_{t}^{0}\right\}\right\|_{L^{q}} & \lesssim\left\{\int_{\frac{|t|}{2}}^{+\infty} d r(\sinh r)^{n-1} \varphi_{0}(r)\left|w_{t}^{0}(r)\right|^{\frac{q}{2}}\right\}^{\frac{2}{q}}\|f\|_{L^{q^{\prime}}} \\
& \lesssim \underbrace{\left\{\int_{\frac{|t|}{2}}^{+\infty} d r r^{\frac{q}{2}+1} e^{-\left(\frac{q}{2}-1\right) \rho r}\right\}^{\frac{2}{q}}\|f\|_{L^{q^{\prime}}} \quad \forall f \in L^{q^{\prime}} .}_{\lesssim|t|^{-\infty}}
\end{aligned}
$$

Estimate 3: We proceed by interpolation for the analytic family (34). If $\operatorname{Re} \sigma=0$, then

$$
\left\|f * \widetilde{w}_{t}^{\infty}\right\|_{L^{2}} \lesssim\|f\|_{L^{2}} \quad \forall f \in L^{2} .
$$

If $\operatorname{Re} \sigma=\frac{n+1}{2}$, we deduce from Theorem 3.2, i that

$$
\left\|f * \widetilde{w}_{t}^{\infty}\right\|_{L^{\infty}} \lesssim|t|^{-\infty}\|f\|_{L^{1}} \quad \forall f \in L^{1} .
$$

By interpolation we obtain for $\sigma=(n+1)\left(\frac{1}{2}-\frac{1}{q}\right)$ that

$$
\left\|f * w_{t}^{\infty}\right\|_{L^{q}} \lesssim|t|^{-\infty}\|f\|_{L^{q^{\prime}}} \quad \forall f \in L^{q^{\prime}} .
$$

We conclude the proof of Theorem 4.3 by summing up the previous estimates.

\subsection{Global dispersive estimates.}

As noticed in Remark 3.4, similar results hold for the operators $D^{-\sigma} \widetilde{D}^{-\tilde{\sigma}} e^{i t D}$.

Corollary 4.4. Let $2<q<\infty$ and $\sigma, \tilde{\sigma} \in \mathbb{R}$ such that $\sigma+\tilde{\sigma} \geq(n+1)\left(\frac{1}{2}-\frac{1}{q}\right)$. Then

$$
\left\|D^{-\sigma} \widetilde{D}^{-\tilde{\sigma}} e^{i t D}\right\|_{L^{q^{\prime} \rightarrow L^{q}}} \lesssim \begin{cases}|t|^{-(n-1)\left(\frac{1}{2}-\frac{1}{q}\right)} & \text { if } 0<|t| \leq 1 \\ |t|^{-\frac{3}{2}} & \text { if }|t| \geq 1\end{cases}
$$

In particular, if $2<q<\infty$ and $\sigma \geq(n+1)\left(\frac{1}{2}-\frac{1}{q}\right)$, then

$$
\left\|\widetilde{D}^{-\sigma} e^{i t D}\right\|_{L^{q^{\prime} \rightarrow L^{q}}}+\left\|\widetilde{D}^{1-\sigma} \frac{e^{i t D}}{D}\right\|_{L^{q^{\prime} \rightarrow L^{q}}} \lesssim \begin{cases}|t|^{-(n-1)\left(\frac{1}{2}-\frac{1}{q}\right)} & \text { if } 0<|t| \leq 1, \\ |t|^{-\frac{3}{2}} & \text { if }|t| \geq 1 .\end{cases}
$$

These results hold in dimension $n \geq 3$. In dimension $n=2$, there is an additional logarithmic factor in the small time bound, which reads $|t|^{-\left(\frac{1}{2}-\frac{1}{q}\right)}(1-\log |t|)^{1-\frac{2}{q}}$.

Remark 4.5. On $L^{2}\left(\mathbb{H}^{n}\right)$, we know by spectral theory that 
- $e^{i t D}$ is a one-parameter group of unitary operators,

- $D^{-\sigma} \tilde{D}^{-\tilde{\sigma}}$ is a bounded operator if $\sigma+\tilde{\sigma} \geq 0$.

Remark 4.6. Let us specialize our results for the wave equation (6). In this case, we have $D=\sqrt{-\Delta}$ and we may take $\tilde{D}=D$. Let $2<q<\infty$ and $\sigma \geq(n+1)\left(\frac{1}{2}-\frac{1}{q}\right)$. Then

$$
\left\|D^{-\sigma} e^{i t D}\right\|_{L^{q^{\prime} \rightarrow L^{q}}} \lesssim \begin{cases}|t|^{-(n-1)\left(\frac{1}{2}-\frac{1}{q}\right)} & \text { if } 0<|t| \leq 1 \\ |t|^{-\frac{3}{2}} & \text { if }|t| \geq 1\end{cases}
$$

in dimension $n \geq 3$ and

$$
\left\|D^{-\sigma} e^{i t D}\right\|_{L^{q^{\prime} \rightarrow L^{q}}} \lesssim \begin{cases}|t|^{-\left(\frac{1}{2}-\frac{1}{q}\right)}(1-\log |t|)^{1-\frac{2}{q}} & \text { if } 0<|t| \leq 1 \\ |t|^{-\frac{3}{2}} & \text { if }|t| \geq 1\end{cases}
$$

in dimension $n=2$. Let us compare (37) with the dispersive estimates obtained by Metcalfe and Taylor [27, Section 3] in dimension $n=3$. Our results are the same when $|t|$ is small and $2<q<\infty$ or when $|t|$ is large and $4 \leq q<\infty$. But our bound $|t|^{-\frac{3}{2}}$ is better than their bound $|t|^{-6\left(\frac{1}{2}-\frac{1}{q}\right)}$ when $|t|$ is large and $2<q<4$. On the other hand, they are able to deal with the endpoint case $q=\infty$, using local Hardy and BMO spaces on $\mathbb{H}^{n}$. 


\section{Strichartz estimates}

We shall assume $n \geq 4$ throughout this section and discuss the dimensions $n=3$ and $n=2$ in the final remarks. Consider the linear equation (12) on $\mathbb{H}^{n}$, whose solution is given by Duhamel's formula:

$$
u(t, x)=\underbrace{\left(\cos t D_{x}\right) f(x)+\frac{\sin t D_{x}}{D_{x}} g(x)}_{u_{\mathrm{hom}}(t, x)}+\underbrace{\int_{0}^{t} d s \frac{\sin (t-s) D_{x}}{D_{x}} F(s, x)}_{u_{\text {inhom }}(t, x)} .
$$

In Appendix B, we recall the definition of Sobolev spaces on $\mathbb{H}^{n}$ and collect some of their properties.

Definition 5.1. A couple $(p, q)$ will be called admissible if $\left(\frac{1}{p}, \frac{1}{q}\right)$ belongs to the triangle

$$
\left\{\left(\frac{1}{p}, \frac{1}{q}\right) \in\left(0, \frac{1}{2}\right] \times\left(0, \frac{1}{2}\right) \mid \frac{1}{p} \geq \frac{n-1}{2}\left(\frac{1}{2}-\frac{1}{q}\right)\right\} \cup\left\{\left(0, \frac{1}{2}\right)\right\} .
$$

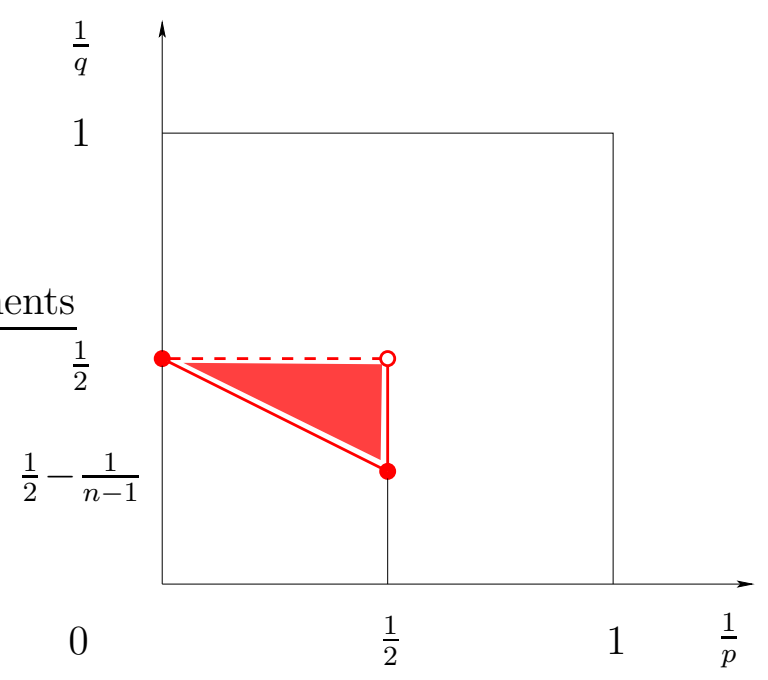

FiguRE 1. Admissibility in dimension $n \geq 4$

Theorem 5.2. Let $(p, q)$ and $(\tilde{p}, \tilde{q})$ be two admissible couples, and let

$$
\sigma \geq \frac{(n+1)}{2}\left(\frac{1}{2}-\frac{1}{q}\right) \quad \text { and } \quad \tilde{\sigma} \geq \frac{(n+1)}{2}\left(\frac{1}{2}-\frac{1}{\tilde{q}}\right) \text {. }
$$

Then the following Strichartz estimate holds for solutions to the Cauchy problem (12):

$$
\left\|\nabla_{\mathbb{R} \times \mathbb{H}^{n}} u\right\|_{L^{p} H^{-\sigma, q}} \lesssim\|f\|_{H^{1}}+\|g\|_{L^{2}}+\|F\|_{L^{\tilde{p}^{\prime} H^{\tilde{\sigma}, \tilde{q}^{\prime}}}} .
$$

Proof. We shall prove the following estimate, which amounts to (40) :

$$
\begin{aligned}
& \left\|\tilde{D}_{x}^{-\sigma+1 / 2} u(t, x)\right\|_{L_{t}^{p} L_{x}^{q}}+\left\|\tilde{D}_{x}^{-\sigma-1 / 2} \partial_{t} u(t, x)\right\|_{L_{t}^{p} L_{x}^{q}} \\
& \lesssim\left\|D_{x}^{1 / 2} f(x)\right\|_{L_{x}^{2}}+\left\|D_{x}^{-1 / 2} g(x)\right\|_{L_{x}^{2}}+\left\|\tilde{D}_{x}^{\tilde{\sigma}-1 / 2} F(t, x)\right\|_{L_{t}^{\tilde{p}^{\prime}} L_{x}^{\tilde{q}^{\prime}}} .
\end{aligned}
$$

Consider the operator

$$
T f(t, x)=\tilde{D}_{x}^{-\sigma+1 / 2} \frac{e^{ \pm i t D_{x}}}{\sqrt{D_{x}}} f(x),
$$


initially defined from $L^{2}\left(\mathbb{H}^{n}\right)$ into $L^{\infty}\left(\mathbb{R} ; L^{2}\left(\mathbb{H}^{n}\right)\right)$, and its formal adjoint

$$
T^{*} F(x)=\int_{-\infty}^{+\infty} d s \tilde{D}_{x}^{-\sigma+1 / 2} \frac{e^{\mp i s D_{x}}}{\sqrt{D_{x}}} F(s, x),
$$

initially defined from $L^{1}\left(\mathbb{R} ; L^{2}\left(\mathbb{H}^{n}\right)\right)$ into $L^{2}\left(\mathbb{H}^{n}\right)$. The $T T^{*}$ method consists in proving first the $L^{p^{\prime}}\left(\mathbb{R} ; L^{q^{\prime}}\left(\mathbb{H}^{n}\right)\right) \rightarrow L^{p}\left(\mathbb{R} ; L^{q}\left(\mathbb{H}^{n}\right)\right)$ boundedness of the operator

$$
T T^{*} F(t, x)=\int_{-\infty}^{+\infty} d s \tilde{D}_{x}^{-2 \sigma+1} \frac{e^{ \pm i(t-s) D_{x}}}{D_{x}} F(s, x)
$$

and of its truncated version

$$
\mathcal{T} F(t, x)=\int_{-\infty}^{t} d s \tilde{D}_{x}^{-2 \sigma+1} \frac{e^{ \pm i(t-s) D_{x}}}{D_{x}} F(s, x),
$$

for every admissible couple $(p, q)$ and for every $\sigma \geq \frac{n+1}{2}\left(\frac{1}{2}-\frac{1}{q}\right)$, and in decoupling next the indices.

We may disregard the endpoint case $(p, q)=(\infty, 2)$, which is easily dealt with, using the boundedness on $L^{2}\left(\mathbb{H}^{n}\right)$ of $e^{i t D}(t \in \mathbb{R})$ and $\tilde{D}^{-\sigma+\frac{1}{2}} D^{-\frac{1}{2}}(\sigma \geq 0)$. Thus assume that $(p, q)$ is an admissible couple, which is different from the endpoints $(\infty, 2)$ and $\left(2,2 \frac{n-1}{n-3}\right)$. It follows from (36) that the norms $\left\|T T^{*} F(t, x)\right\|_{L_{t}^{p} L_{x}^{q}}$ and $\|\mathcal{T} F(t, x)\|_{L_{t}^{p} L_{x}^{q}}$ are bounded above by

$$
\left\|\int_{0<|t-s|<1} d s|t-s|^{-\alpha}\right\| F(s, x)\left\|_{L_{x}^{q^{\prime}}}\right\|_{L_{t}^{p}}+\left\|\int_{|t-s| \geq 1} d s|t-s|^{-\frac{3}{2}}\right\| F(s, x)\left\|_{L_{x}^{q^{\prime}}}\right\|_{L_{t}^{p}},
$$

where $\alpha=(n-1)\left(\frac{1}{2}-\frac{1}{q}\right) \in(0,1)$. On one hand, the convolution kernel $|t-s|^{-\frac{3}{2}} \mathbb{I}_{\{|t-s| \geq 1\}}$ defines obviously a bounded operator from $L^{p_{1}}(\mathbb{R})$ to $L^{p_{2}}(\mathbb{R})$, for all $1 \leq p_{1} \leq p_{2} \leq \infty$, in particular from $L^{p^{\prime}}(\mathbb{R})$ to $L^{p}(\mathbb{R})$, since $p \geq 2$. On the other hand, the convolution kernel $|t-s|^{-\alpha} \mathbb{I}_{\{0<|t-s| \leq 1\}}$ with $0<\alpha<1$ defines a bounded operator from $L^{p_{1}}(\mathbb{R})$ to $L^{p_{2}}(\mathbb{R})$, for all $1<p_{1}, p_{2}<\infty$ such that $0 \leq \frac{1}{p_{1}}-\frac{1}{p_{2}} \leq 1-\alpha$, in particular from $L^{p^{\prime}}(\mathbb{R})$ to $L^{p}(\mathbb{R})$, since $p \geq 2$ and $\frac{2}{p} \geq \alpha$.

At the endpoint $(p, q)=\left(2,2 \frac{n-1}{n-3}\right)$, we have $\alpha=1$. Thus the previous argument breaks down and is replaced by the refined analysis carried out in [22]. Notice that the problem lies only in the first part of (42) and not in the second one, which involves an integrable convolution kernel on $\mathbb{R}$.

Thus $T T^{*}$ and $\mathcal{T}$ are bounded from $L^{p^{\prime}}\left(\mathbb{R} ; L^{q^{\prime}}\left(\mathbb{H}^{n}\right)\right)$ to $L^{p}\left(\mathbb{R} ; L^{q}\left(\mathbb{H}^{n}\right)\right)$, for every admissible couple $(p, q)$. As a consequence, $T^{*}$ is bounded from $L^{p^{\prime}}\left(\mathbb{R} ; L^{q^{\prime}}\left(\mathbb{H}^{n}\right)\right)$ to $L^{2}\left(\mathbb{H}^{n}\right)$ and $T$ is bounded from $L^{2}\left(\mathbb{H}^{n}\right)$ to $L^{p}\left(\mathbb{R} ; L^{q}\left(\mathbb{H}^{n}\right)\right)$. We deduce in particular that

$$
\left\|\tilde{D}_{x}^{-\sigma+1 / 2}\left(\cos t D_{x}\right) f(x)\right\|_{L_{t}^{p} L_{x}^{q}} \lesssim\left\|\tilde{D}_{x}^{-\sigma+1 / 2} e^{ \pm i t D_{x}} f(x)\right\|_{L_{t}^{p} L_{x}^{q}} \lesssim\left\|D_{x}^{1 / 2} f(x)\right\|_{L_{x}^{2}}
$$

and

$$
\left\|\tilde{D}_{x}^{-\sigma+1 / 2} \frac{\sin t D_{x}}{D_{x}} g(x)\right\|_{L_{t}^{p} L_{x}^{q}} \lesssim\left\|\tilde{D}_{x}^{-\sigma+1 / 2} D_{x}^{-1} e^{ \pm i t D_{x}} g(x)\right\|_{L_{t}^{p} L_{x}^{q}} \lesssim\left\|D_{x}^{-1 / 2} g(x)\right\|_{L_{x}^{2}} .
$$


In summary,

$$
\left\|\tilde{D}_{x}^{-\sigma+1 / 2} u_{\mathrm{hom}}(t, x)\right\|_{L_{t}^{p} L_{x}^{q}} \lesssim\left\|D_{x}^{1 / 2} f(x)\right\|_{L_{x}^{2}}+\left\|D_{x}^{-1 / 2} g(x)\right\|_{L_{x}^{2}} .
$$

We next decouple the indices in the $L^{p^{\prime}} L^{q^{\prime}} \rightarrow L^{q} L^{q}$ estimate of $T T^{*}$ and $\mathcal{T}$. Let $(p, q) \neq(\tilde{p}, \tilde{q})$ be two admissible couples and let $\sigma \geq \frac{n+1}{2}\left(\frac{1}{2}-\frac{1}{q}\right), \tilde{\sigma} \geq \frac{n+1}{2}\left(\frac{1}{2}-\frac{1}{\tilde{q}}\right)$. Since $T$ and $T^{*}$ are separately continuous, the operator

$$
T T^{*} F(t, x)=\int_{-\infty}^{+\infty} d s \tilde{D}_{x}^{-\sigma-\tilde{\sigma}+1} \frac{e^{ \pm i(t-s) D_{x}}}{D_{x}} F(s, x)
$$

is bounded from $L^{\tilde{p}^{\prime}}\left(\mathbb{R} ; L^{\tilde{q}^{\prime}}\left(\mathbb{H}^{n}\right)\right)$ to $L^{p}\left(\mathbb{R} ; L^{q}\left(\mathbb{H}^{n}\right)\right)$. According to [5], this result remains true for the truncated operator

$$
\mathcal{T} F(t, x)=\int_{-\infty}^{t} d s \tilde{D}_{x}^{-\sigma-\tilde{\sigma}+1} \frac{e^{ \pm i(t-s) D_{x}}}{D_{x}} F(s, x)
$$

and hence for

$$
\widetilde{\mathcal{T}} F(t, x)=\int_{0}^{t} d s \tilde{D}_{x}^{-\sigma-\tilde{\sigma}+1} \frac{\sin (t-s) D_{x}}{D_{x}} F(s, x)
$$

as long as $p$ and $\tilde{p}$ are not both equal to 2 . For the remaining case, where $p=\tilde{p}=2$ and $2<q \neq \tilde{q} \leq 2 \frac{n-1}{n-3}$, we argue as in the proof of [2, Theorem 6.3] by resuming part of the bilinear approach in [22]. Hence

$$
\left\|\tilde{D}_{x}^{-\sigma+1 / 2} u_{\text {inhom }}(t, x)\right\|_{L_{t}^{p} L_{x}^{q}} \lesssim\left\|\tilde{D}_{x}^{\tilde{\sigma}-1 / 2} F(t, x)\right\|_{L_{t}^{\tilde{p}^{\prime}} L_{x}^{\tilde{q}^{\prime}}}
$$

for all admissible couples $(p, q)$ and $(\tilde{p}, \tilde{q})$.

The Strichartz estimate

$$
\left\|\tilde{D}_{x}^{-\sigma+1 / 2} u(t, x)\right\|_{L_{t}^{p} L_{x}^{q}} \lesssim\left\|D_{x}^{1 / 2} f(x)\right\|_{L_{x}^{2}}+\left\|D_{x}^{-1 / 2} g(x)\right\|_{L_{x}^{2}}+\left\|\tilde{D}_{x}^{\tilde{\sigma}-1 / 2} F(t, x)\right\|_{L_{t}^{\tilde{p}^{\prime}} L_{x}^{\tilde{q}^{\prime}}}
$$

is obtained by summing up the homogeneous estimate (43) and the inhomogeneous estimate (44). As far as it is concerned, the Strichartz estimate of

$$
\partial_{t} u(t, x)=-\left(\sin t D_{x}\right) D_{x} f(x)+\left(\cos t D_{x}\right) g(x)+\int_{0}^{t} d s\left[\cos (t-s) D_{x}\right] F(s, x)
$$

is obtained in the same way and is actually easier. More precisely, we consider this time the operator

and its adjoint

$$
\widetilde{T} f(t, x)=\tilde{D}_{x}^{-\sigma} e^{ \pm i t D_{x}} f(x),
$$

$$
\widetilde{T}^{*} F(x)=\int_{-\infty}^{+\infty} d s \tilde{D}_{x}^{-\sigma} e^{\mp i s D_{x}} F(s, x) .
$$

By using the Sobolev embedding theorem, Theorem 5.2 can be extended to all couples $\left(\frac{1}{p}, \frac{1}{q}\right)$ and $\left(\frac{1}{\tilde{p}}, \frac{1}{\tilde{q}}\right)$ in the square

$$
\left[0, \frac{1}{2}\right] \times\left(0, \frac{1}{2}\right) \cup\left\{\left(0, \frac{1}{2}\right)\right\}
$$


Corollary 5.3. Let $(p, q),(\tilde{p}, \tilde{q})$ be two couples corresponding to the square (45) and let $\sigma, \tilde{\sigma} \in \mathbb{R}$. Assume that $\sigma \geq \sigma(p, q)$, where

$$
\sigma(p, q)=\frac{n+1}{2}\left(\frac{1}{2}-\frac{1}{q}\right)+\max \left\{0, \frac{n-1}{2}\left(\frac{1}{2}-\frac{1}{q}\right)-\frac{1}{p}\right\}= \begin{cases}\frac{n+1}{2}\left(\frac{1}{2}-\frac{1}{q}\right) & \text { if } \frac{1}{p} \geq \frac{n-1}{2}\left(\frac{1}{2}-\frac{1}{q}\right), \\ n\left(\frac{1}{2}-\frac{1}{q}\right)-\frac{1}{p} & \text { if } \frac{1}{p} \leq \frac{n-1}{2}\left(\frac{1}{2}-\frac{1}{q}\right),\end{cases}
$$

and similarly $\tilde{\sigma} \geq \sigma(\tilde{p}, \tilde{q})$. Then the conclusion of Theorem5.2 holds for solutions to the Cauchy problem (12). More precisely we have again the Strichartz estimate

(40)

$$
\left\|\nabla_{\mathbb{R} \times \mathbb{H}^{n}} u\right\|_{L^{p} H^{-\sigma, q}} \lesssim\|f\|_{H^{1}}+\|g\|_{L^{2}}+\|F\|_{L^{\tilde{p}^{\prime}} H^{\tilde{\sigma} \tilde{q}^{\prime}}}
$$

which amounts to

$$
\begin{aligned}
& \left\|\tilde{D}_{x}^{-\sigma+1 / 2} u(t, x)\right\|_{L_{t}^{p} L_{x}^{q}}+\left\|\tilde{D}_{x}^{-\sigma-1 / 2} \partial_{t} u(t, x)\right\|_{L_{t}^{p} L_{x}^{q}} \\
& \lesssim\left\|D_{x}^{1 / 2} f(x)\right\|_{L_{x}^{2}}+\left\|D_{x}^{-1 / 2} g(x)\right\|_{L_{x}^{2}}+\left\|\tilde{D}_{x}^{\tilde{\sigma}-1 / 2} F(t, x)\right\|_{L_{t}^{\tilde{p}^{\prime}} L_{x}^{\tilde{q}^{\prime}}} \cdot
\end{aligned}
$$

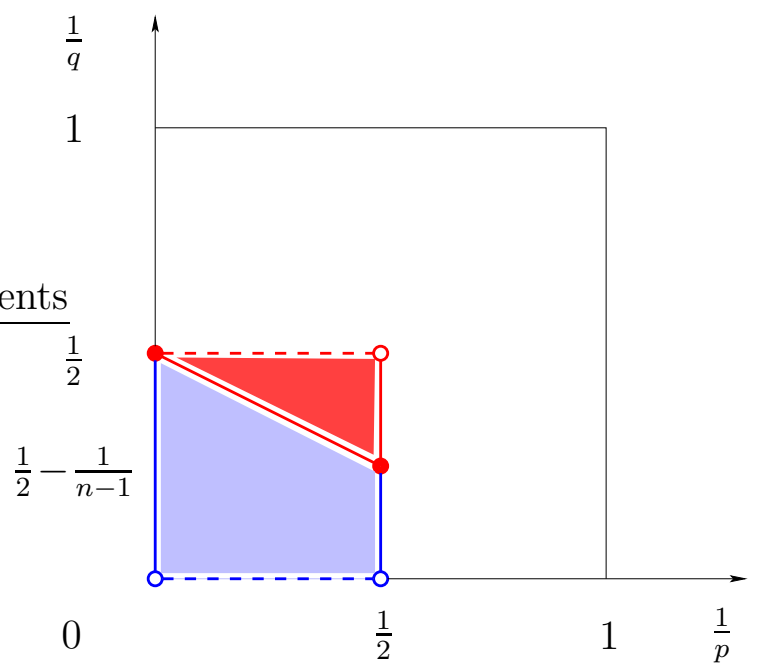

Figure 2. Case $n \geq 4$

Proof. We may retrict to the limit cases $\sigma=\sigma(p, q)$ and $\tilde{\sigma}=\sigma(\tilde{p}, \tilde{q})$. Define $Q$ by

$$
\frac{1}{Q}= \begin{cases}\frac{1}{q} & \text { if } \frac{1}{p} \geq \frac{n-1}{2}\left(\frac{1}{2}-\frac{1}{q}\right), \\ \frac{1}{2}-\frac{2}{n-1} \frac{1}{p} & \text { if } \frac{1}{p} \leq \frac{n-1}{2}\left(\frac{1}{2}-\frac{1}{q}\right) .\end{cases}
$$

and similarly $\tilde{Q}$. Since $(p, Q)$ and $(\tilde{p}, \tilde{Q})$ are admissible couples, it follows from Theorem 5.2 and more precisely from (41) that

$$
\begin{aligned}
& \left\|\tilde{D}_{x}^{-\Sigma+1 / 2} u(t, x)\right\|_{L_{t}^{p} L_{x}^{Q}}+\left\|\tilde{D}_{x}^{-\tilde{\Sigma}-1 / 2} \partial_{t} u(t, x)\right\|_{L_{t}^{p} L_{x}^{Q}} \\
& \lesssim\left\|D_{x}^{1 / 2} f(x)\right\|_{L_{x}^{2}}+\left\|D_{x}^{-1 / 2} g(x)\right\|_{L_{x}^{2}}+\left\|\tilde{D}_{x}^{\tilde{\Sigma}-1 / 2} F(t, x)\right\|_{L_{t}^{\tilde{p}^{\prime}} L_{x}^{\tilde{Q}^{\prime}}}
\end{aligned}
$$


where $\Sigma=\frac{n+1}{2}\left(\frac{1}{2}-\frac{1}{Q}\right)$ and $\tilde{\Sigma}=\frac{n+1}{2}\left(\frac{1}{2}-\frac{1}{\tilde{Q}}\right)$. Since $\sigma-\Sigma=n\left(\frac{1}{Q}-\frac{1}{q}\right)$, we have

$$
\left\|\tilde{D}_{x}^{-\sigma+1 / 2} u(t, x)\right\|_{L_{t}^{p} L_{x}^{q}} \lesssim\left\|\tilde{D}_{x}^{-\Sigma+1 / 2} u(t, x)\right\|_{L_{t}^{p} L_{x}^{Q}}
$$

according to the Sobolev embedding theorem (Proposition B.1). Similarly,

$$
\left\|\tilde{D}_{x}^{\tilde{\Sigma}-1 / 2} F(t, x)\right\|_{L_{t}^{\tilde{p}^{\prime}} L_{x}^{\tilde{Q}^{\prime}}} \lesssim\left\|\tilde{D}_{x}^{\tilde{\sigma}-1 / 2} F(t, x)\right\|_{L_{t}^{\tilde{p}^{\prime}} L_{x}^{\tilde{q}}}
$$

We conclude by combining (46), (47), (48),

Remark 5.4. Theorem 5.2 and Corollary 5.3 hold true in dimension $n=3$ with the same proofs. Notice that the endpoint $(p, q)=(2, \infty)$ is excluded. These results hold in particular for the 3D wave equation (6) and include the Strichartz estimates obtained by Metcalfe and Taylor [27, Section 4] in the smaller region

$$
\left\{\left(\frac{1}{p}, \frac{1}{q}\right) \in\left[0, \frac{1}{2}\right] \times\left(0, \frac{1}{2}\right] \mid \frac{1}{p} \leq 3\left(\frac{1}{2}-\frac{1}{q}\right)\right\} \backslash\left\{\left(\frac{1}{2}, \frac{1}{3}\right)\right\} .
$$

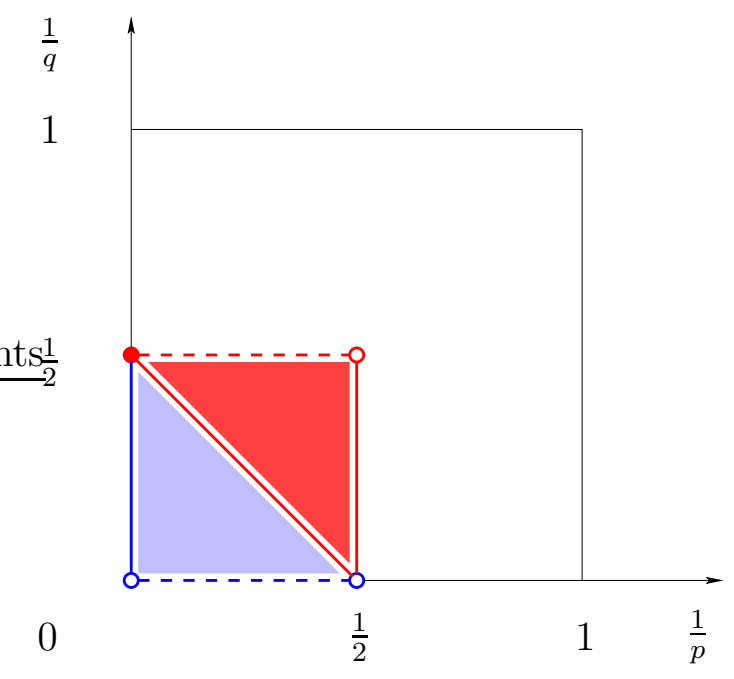

Figure 3. Case $n=3$

Remark 5.5. The analysis carried out in this section still holds in dimension $n=2$, except for the first convolution kernel in (42), which becomes

$$
|t-s|^{-\alpha}(1-\log |t-s|)^{\beta} \mathbb{I}_{\{0<|t-s|<1\}},
$$

with $\alpha=\frac{1}{2}-\frac{1}{q}$ and $\beta=2\left(\frac{1}{2}-\frac{1}{q}\right)$. Consequently, the admissibility region in Theorem 5.2 becomes

$$
\left\{\left(\frac{1}{p}, \frac{1}{q}\right) \in\left(0, \frac{1}{2}\right] \times\left(0, \frac{1}{2}\right) \mid \frac{1}{p}>\frac{1}{2}\left(\frac{1}{2}-\frac{1}{q}\right)\right\} \cup\left\{\left(0, \frac{1}{2}\right)\right\}
$$

and the inequality $\sigma \geq \sigma(p, q)$, resp. $\tilde{\sigma} \geq \sigma(\tilde{p}, \tilde{q})$ in Corollary 5.3 becomes strict in the triangle

$$
\left\{\left(\frac{1}{p}, \frac{1}{q}\right) \in\left(0, \frac{1}{4}\right) \times\left(0, \frac{1}{2}\right) \mid \frac{1}{p} \leq \frac{1}{2}\left(\frac{1}{2}-\frac{1}{q}\right)\right\} .
$$




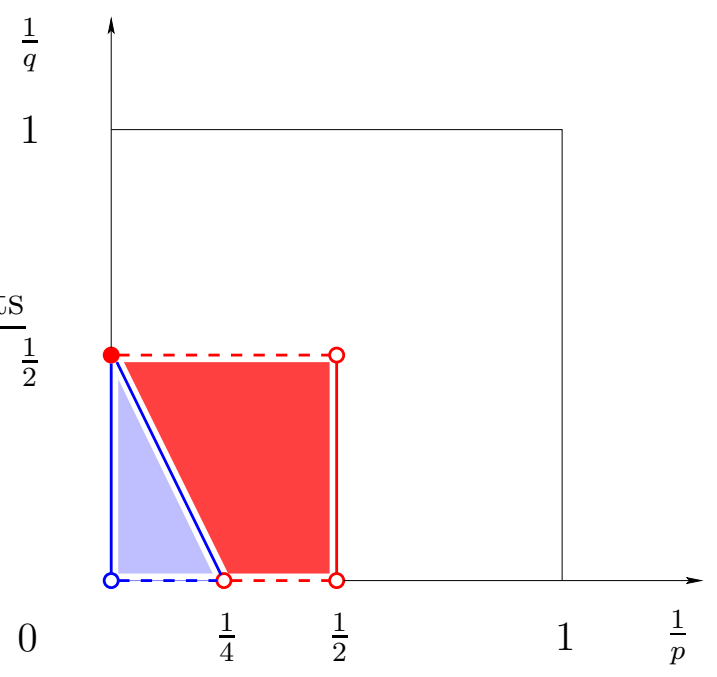

Figure 4 . Case $n=2$

\section{Global WelL-POSEDness In $L^{p}\left(\mathbb{R}, L^{q}\left(\mathbb{H}^{n}\right)\right)$}

In this section, following the classical fixed point scheme, we use the Strichartz estimates obtained in Section 5 to prove global well-posedness for the semilinear equation

$$
\left\{\begin{array}{l}
\partial_{t}^{2} u(t, x)+D_{x}^{2} u(t, x)=F(u(t, x)) \\
u(0, x)=f(x),\left.\partial_{t}\right|_{t=0} u(t, x)=g(x)
\end{array}\right.
$$

on $\mathbb{H}^{n}$ with power-like nonlinearities

$$
F(u) \sim|u|^{\gamma} \quad(\gamma>1)
$$

and small initial data $f$ and $g$. We assume $n \geq 3$ throughout the section and discuss the 2 -dimensional case in the final remark. The statement and proof of our result involve the following powers

$$
\begin{gathered}
\gamma_{1}=1+\frac{3}{n}, \quad \gamma_{2}=1+\frac{2}{\frac{n-1}{2}+\frac{2}{n-1}}, \quad \gamma_{\text {conf }}=1+\frac{4}{n-1}, \\
\gamma_{3}= \begin{cases}\frac{\frac{n+6}{2}+\frac{2}{n-1}+\sqrt{4 n+\left(\frac{6-n}{2}+\frac{2}{n-1}\right)^{2}}}{n} & \text { if } n \leq 5, \\
1+\frac{2}{\frac{n-1}{2}-\frac{1}{n-1}} & \text { if } n \geq 6,\end{cases} \\
\gamma_{4}= \begin{cases}1+\frac{4}{n-2} & \text { if } n \leq 5, \\
\frac{n-1}{2}+\frac{3}{n+1}-\sqrt{\left(\frac{n-3}{2}+\frac{3}{n+1}\right)^{2}-4 \frac{n-1}{n+1}} & \text { if } n \geq 6,\end{cases}
\end{gathered}
$$

and the following curves

$$
\sigma_{1}(\gamma)=\frac{n+1}{4}-\frac{(n+1)(n+5)}{8 n} \frac{1}{\gamma-\frac{n+1}{2 n}}, \quad \sigma_{2}(\gamma)=\frac{n+1}{4}-\frac{1}{\gamma-1}, \quad \sigma_{3}(\gamma)=\frac{n}{2}-\frac{2}{\gamma-1} .
$$




\begin{tabular}{|c|c|c|c|c|c|}
\hline$n$ & $\gamma_{1}$ & $\gamma_{2}$ & $\gamma_{\text {conf }}$ & $\gamma_{3}$ & $\gamma_{4}$ \\
\hline 3 & 2 & 2 & 3 & $\frac{11+\sqrt{73}}{6} \simeq 3,26$ & 5 \\
\hline 4 & $\frac{7}{4}=1,75$ & $\frac{25}{13} \simeq 1,92$ & $\frac{7}{3} \simeq 2,33$ & $\frac{5}{2} \simeq 2,5$ & 3 \\
\hline 5 & $\frac{8}{5} \simeq 1,6$ & $\frac{9}{5} \simeq 1,8$ & 2 & $\frac{6+\sqrt{21}}{5} \simeq 2,12$ & $\frac{7}{3} \simeq 2,33$ \\
\hline 6 & $\frac{3}{2}=1,5$ & $\frac{49}{29} \simeq 1,69$ & $\frac{9}{5}=1,8$ & $\frac{43}{23} \simeq 1,87$ & 2 \\
\hline$\geq 7$ & $<\gamma_{2}$ & $<\gamma_{\text {conf }}$ & $<\gamma_{3}$ & $<\gamma_{4}$ & $<2$ \\
\hline
\end{tabular}

The powers $\gamma_{1}, \gamma_{2}, \gamma_{\text {conf }}$ and the curves $C_{1}, C_{2}, C_{3}$ parametrized by $\sigma_{1}, \sigma_{2}, \sigma_{3}$ occur already in the Euclidean setting. More precisely, they are involved in the conditions, illustrated in Figure 5, of minimal regularity $\sigma$ on the initial data $f, g$ which are needed in order to ensure local well-posedness of (49). We refer again to [21, 25, 22 for more details. Notice that, in dimension $n=3, \gamma_{1}$ coincides with $\gamma_{2}$ and there is no curve $C_{1}$.

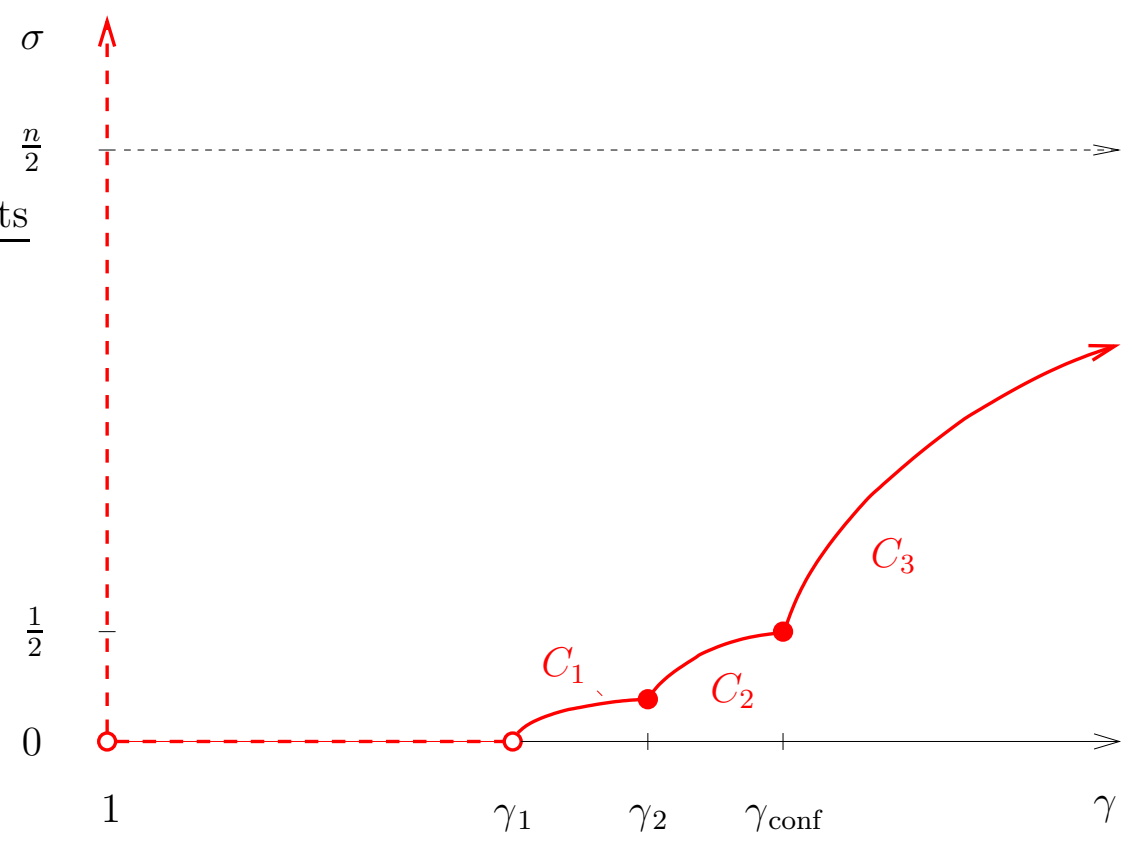

FiguRE 5. Regularity for LWP on $\mathbb{R}^{n}$ in dimension $n \geq 3$

As mentionned in the introduction, global well-posedness of (49) on $\mathbb{R}^{n}$ requires additional conditions. Recall that smooth solutions with small amplitude blow up or not depending whether $\gamma$ is smaller or larger than the critical power $\gamma_{0}$ defined in (5).

In Section 5 we have obtained Strichartz estimates on $\mathbb{H}^{n}$ for a range of admissible couples which is wider than on $\mathbb{R}^{n}$. As a consequence, we deduce in this section stronger well-posedness results for (49). In particular, we prove global well-posedness for small initial data in $H^{\sigma}\left(\mathbb{H}^{n}\right) \times H^{\sigma-1}\left(\mathbb{H}^{n}\right)$, if $1<\gamma<\gamma_{1}$ and $\sigma>0$ is small. Thus there is no blow-up for small powers $\gamma>1$ on $\mathbb{H}^{n}$, in sharp contrast with $\mathbb{R}^{n}$. 
Theorem 6.1. Assume that the nonlinearity F satisfies

$$
|F(u)| \leq C|u|^{\gamma}, \quad|F(u)-F(v)| \leq C\left(|u|^{\gamma-1}+|v|^{\gamma-1}\right)|u-v| .
$$

Then, in dimension $n \geq 3$, the equation (49) is globally well-posed for small initial data in $H^{\sigma}\left(\mathbb{H}^{n}\right) \times H^{\sigma-1}\left(\mathbb{H}^{n}\right)$ provided

$$
\begin{cases}\sigma=0^{+} & \text {if } 1<\gamma \leq \gamma_{1} \\ \sigma=\sigma_{1}(\gamma) & \text { if } \gamma_{1}<\gamma \leq \gamma_{2}, \\ \sigma=\sigma_{2}(\gamma) & \text { if } \gamma_{2} \leq \gamma \leq \gamma_{\mathrm{conf}} \\ \sigma=\sigma_{3}(\gamma) & \text { if } \quad \gamma_{\mathrm{conf}} \leq \gamma \leq \gamma_{4}\end{cases}
$$

where $\sigma=0^{+}$stands for any $\sigma>0$ sufficiently close to 0 . More precisely, in each case, there exist $2 \leq p, q<\infty$ and $\delta, \varepsilon>0$ such that, for any initial data $(f, g) \in$ $H^{\sigma}\left(\mathbb{H}^{n}\right) \times H^{\sigma-1}\left(\mathbb{H}^{n}\right)$ with norm $\leq \delta$, the Cauchy problem (49) has a unique solution $u$ with norm $\leq \varepsilon$ in the Banach space

$$
X=C\left(\mathbb{R} ; H^{\sigma}\left(\mathbb{H}^{n}\right)\right) \cap C^{1}\left(\mathbb{R} ; H^{\sigma-1}\left(\mathbb{H}^{n}\right)\right) \cap L^{p}\left(\mathbb{R} ; L^{q}\left(\mathbb{H}^{n}\right)\right) .
$$

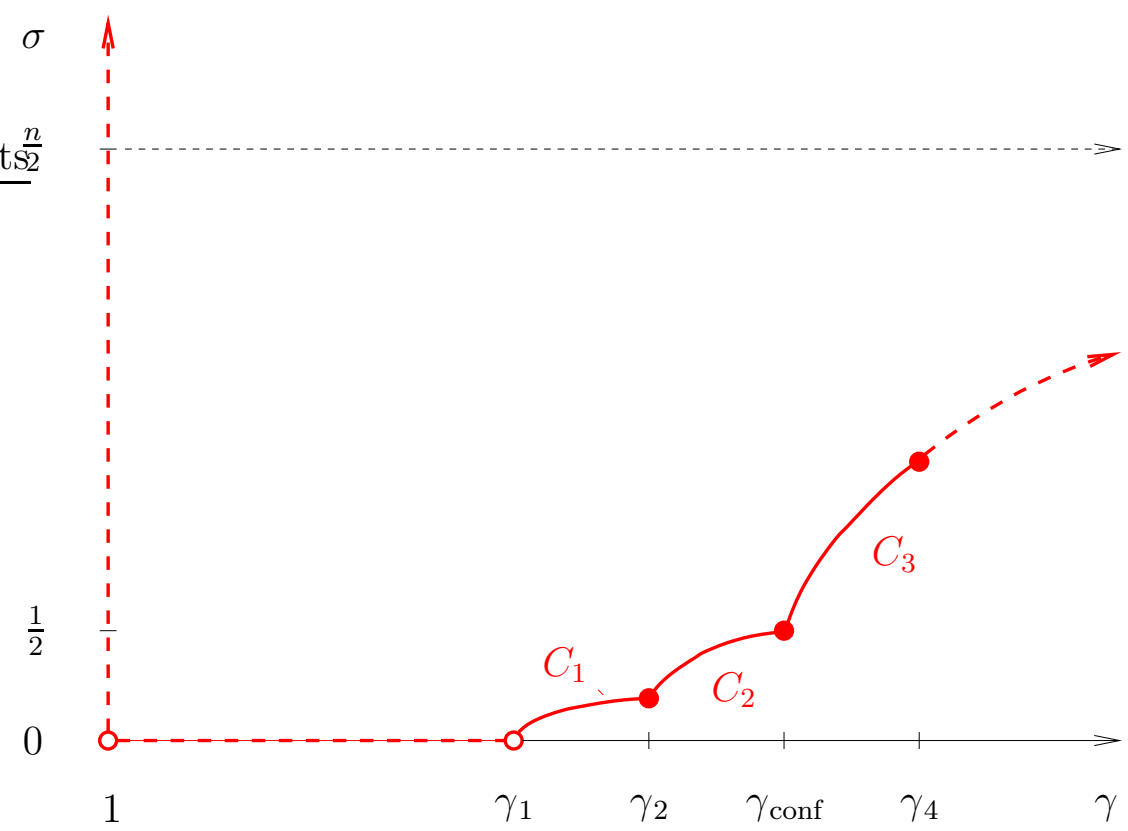

FiguRE 6. Regularity for GWP on $\mathbb{H}^{n}$ in dimension $n \geq 3$

Remark 6.2. In dimension $n=3, \gamma_{1}$ coincides with $\gamma_{2}$, the second and third conditions in (53) boil down to

$$
\sigma \geq \sigma_{2}(\gamma) \quad \text { if } \gamma_{1}=\gamma_{2}<\gamma \leq \gamma_{\mathrm{conf}}
$$

and there is no curve $C_{1}$ in Figure 6. 
Proof of Theorem 6.1 for $1<\gamma \leq \gamma_{\text {conf }}$. We resume the fixed point method based on Strichartz estimates. Define $u=\Phi(v)$ as the solution to the Cauchy problem

$$
\left\{\begin{array}{l}
\partial_{t}^{2} u(t, x)-D_{x}^{2} u(t, x)=F(v(t, x)), \\
u(0, x)=f(x),\left.\partial_{t}\right|_{t=0} u(t, x)=g(x),
\end{array}\right.
$$

which is given by Duhamel's formula:

$$
u(t, x)=\left(\cos t D_{x}\right) f(x)+\frac{\sin t D_{x}}{D_{x}} g(x)+\int_{0}^{t} d s \frac{\sin (t-s) D_{x}}{D_{x}} F(s, x) .
$$

On one hand, according to Theorem 5.2 , the Strichartz estimate

$$
\begin{aligned}
& \|u(t, x)\|_{L_{t}^{\infty} H_{x}^{\sigma}}+\left\|\partial_{t} u(t, x)\right\|_{L_{t}^{\infty} H_{x}^{\sigma-1}}+\|u(t, x)\|_{L_{t}^{p} L_{x}^{q}} \\
& \lesssim\|f(x)\|_{H_{x}^{\sigma}}+\|g(x)\|_{H_{x}^{\sigma-1}}+\|F(v(t, x))\|_{L_{t}^{\tilde{p}^{\prime}} H_{x}^{\sigma+\tilde{\sigma}-1, \tilde{q}^{\prime}}}
\end{aligned}
$$

holds whenever

$$
\left\{\begin{array}{l}
(p, q) \text { and }(\tilde{p}, \tilde{q}) \text { are admissible couples; } \\
\sigma \geq \frac{n+1}{2}\left(\frac{1}{2}-\frac{1}{\tilde{q}}\right) \text { and } \tilde{\sigma} \geq \frac{n+1}{2}\left(\frac{1}{2}-\frac{1}{\tilde{q}}\right) .
\end{array}\right.
$$

On the other hand, by our nonlinear assumption (52) and by the Sobolev embedding theorem (Theorem B.1), we have

$$
\|F(v(t, x))\|_{L_{t}^{\tilde{p}^{\prime}} H_{x}^{\sigma+\tilde{\sigma}-1, \tilde{q}^{\prime}}} \lesssim\left\||v(t, x)|^{\gamma}\right\|_{L_{t}^{\tilde{p}^{\prime}} H_{x}^{\sigma+\tilde{\sigma}-1, \tilde{q}^{\prime}}} \lesssim\left\||v(t, x)|^{\gamma}\right\|_{L_{t}^{\tilde{p}^{\prime}} L_{x}^{\tilde{Q}^{\prime}}} \lesssim\|v(t, x)\|_{L_{t}^{\gamma \tilde{p}^{\prime}} L_{x}^{\gamma \tilde{Q}^{\prime}}}^{\gamma},
$$

provided

$$
\sigma+\tilde{\sigma} \leq 1,1<\tilde{Q}^{\prime} \leq \tilde{q}^{\prime}<\infty \quad \text { and } \quad \frac{n}{\tilde{Q}^{\prime}}-\frac{n}{\tilde{q}^{\prime}} \leq 1-\sigma-\tilde{\sigma} .
$$

In order to remain within the same function space, we require in addition that

$$
\gamma \tilde{p}^{\prime}=p \quad \text { and } \quad \gamma \tilde{Q}^{\prime}=q .
$$

In summary,

$$
\begin{aligned}
& \|u(t, x)\|_{L_{t}^{\infty} H_{x}^{\sigma}}+\left\|\partial_{t} u(t, x)\right\|_{L_{t}^{\infty} H_{x}^{\sigma-1}}+\|u(t, x)\|_{L_{t}^{p} L_{x}^{q}} \\
& \leq C\left\{\|f(x)\|_{H_{x}^{\sigma}}+\|g(x)\|_{H_{x}^{\sigma-1}}+\|v\|_{L_{t}^{p} L_{x}^{q}}^{\gamma}\right\}
\end{aligned}
$$

if the following set of conditions is satisfied:

$$
\left\{\begin{array}{l}
\text { (a) }(p, q) \text { and }(\tilde{p}, \tilde{q}) \text { are admissible couples } \\
\text { (b) } \sigma \geq \frac{n+1}{2}\left(\frac{1}{2}-\frac{1}{q}\right), \tilde{\sigma} \geq \frac{n+1}{2}\left(\frac{1}{2}-\frac{1}{\tilde{q}}\right), \sigma+\tilde{\sigma} \leq 1 \\
\text { (c) } \frac{\gamma}{p}+\frac{1}{\tilde{p}}=1 \\
\text { (d) } 1 \leq \frac{\gamma}{q}+\frac{1}{\tilde{q}} \leq 1+\frac{1-\sigma-\tilde{\sigma}}{n} \\
\text { (e) } q>\gamma
\end{array}\right.
$$

For such a choice, $\Phi$ maps the Banach space

$$
X=C\left(\mathbb{R} ; H^{\sigma}\left(\mathbb{H}^{n}\right)\right) \cap C^{1}\left(\mathbb{R} ; H^{\sigma-1}\left(\mathbb{H}^{n}\right)\right) \cap L^{p}\left(\mathbb{R} ; L^{q}\left(\mathbb{H}^{n}\right)\right),
$$


equipped with the norm

$$
\|u\|_{X}=\|u(t, x)\|_{L_{t}^{\infty} H_{x}^{\sigma}}+\left\|\partial_{t} u(t, x)\right\|_{L_{t}^{\infty} H_{x}^{\sigma-1}}+\|u\|_{L_{t}^{p} L_{x}^{q}}
$$

into itself. Let us show that $\Phi$ is a contraction on the ball

$$
X_{\varepsilon}=\left\{u \in X \mid\|u\|_{X} \leq \varepsilon\right\},
$$

provided $\varepsilon>0$ and $\|f\|_{H^{\sigma}}+\|g\|_{H^{\sigma-1}}$ are sufficiently small. Let $v, \tilde{v} \in X$ and $u=\Phi(v)$, $\tilde{u}=\Phi(\tilde{v})$. By resuming the arguments leading to (56) and by using in addition Hölder's inequality, we obtain the estimate

$$
\begin{aligned}
\|u-\tilde{u}\|_{X} & \leq C\|F(v)-F(\tilde{v})\|_{L_{t}^{\tilde{p^{\prime}}} L_{x}^{\tilde{Q}^{\prime}}} \\
& \leq C\left\|\left\{|v|^{\gamma-1}+|\tilde{v}|^{\gamma-1}\right\}|v-\tilde{v}|\right\|_{L_{t}^{\tilde{p}^{\prime}} L_{x}^{\tilde{Q}^{\prime}}} \\
& \leq C\left\{\|v\|_{L_{t}^{p} L_{x}^{q}}^{\gamma-1}+\|\tilde{v}\|_{L_{t}^{p} L_{x}^{q}}^{\gamma-1}\right\}\|v-\tilde{v}\|_{L_{t}^{p} L_{x}^{q}} \\
& \leq C\left\{\|v\|_{X}^{\gamma-1}+\|\tilde{v}\|_{X}^{\gamma-1}\right\}\|v-\tilde{v}\|_{X} .
\end{aligned}
$$

Thus, if we assume $\|v\|_{X} \leq \varepsilon,\|\tilde{v}\|_{X} \leq \varepsilon$ and $\|f\|_{H^{\sigma}}+\|g\|_{H^{\sigma-1}} \leq \delta$, then (56) and (58) yield

$$
\|u\|_{X} \leq C \delta+C \varepsilon^{\gamma}, \quad\|\tilde{u}\|_{X} \leq C \delta+C \varepsilon^{\gamma} \quad \text { and } \quad\|u-\tilde{u}\|_{X} \leq 2 C \varepsilon^{\gamma-1}\|v-\tilde{v}\|_{X} .
$$

Hence

$$
\|u\|_{X} \leq \varepsilon, \quad\|\tilde{u}\|_{X} \leq \varepsilon \quad \text { and } \quad\|u-\tilde{u}\|_{X} \leq \frac{1}{2}\|v-\tilde{v}\|_{X}
$$

if $C \varepsilon^{\gamma-1} \leq \frac{1}{4}$ and $C \delta \leq \frac{3}{4} \varepsilon$. One concludes by applying the fixed point theorem in the complete metric space $X_{\varepsilon}$.

It remains for us to check that the set of conditions (57) can be fulfilled in the various cases (53). Notice that we may assume the following equalities in (57, b) :

$$
\sigma=\frac{n+1}{2}\left(\frac{1}{2}-\frac{1}{q}\right) \quad \text { and } \quad \tilde{\sigma}=\frac{n+1}{2}\left(\frac{1}{2}-\frac{1}{\tilde{q}}\right) .
$$

Thus (57) reduces to the set of conditions:

$$
\left\{\begin{array}{l}
\text { (a) }(p, q) \text { and }(\tilde{p}, \tilde{q}) \text { are admissible couples } \\
\text { (b) } \frac{1}{q}+\frac{1}{\tilde{q}} \geq \frac{n-1}{n+1} \\
\text { (c) } \frac{\gamma}{p}+\frac{1}{\tilde{\tilde{p}}}=1 \\
\text { (d.i) } \frac{\gamma}{q}+\frac{1}{\tilde{q}} \geq 1 \\
\text { (d.ii) }\left(\frac{2 n}{n-1} \gamma-\frac{n+1}{n-1}\right) \frac{1}{q}+\frac{1}{\tilde{q}} \leq \frac{n+1}{n-1} \\
\text { (e) } q>\gamma
\end{array}\right.
$$

We shall discuss these conditions first in high dimensions and next in low dimensions.

- Assume that $n \geq 6$.

Firstly notice that $\gamma_{\text {conf }}<2$. As $\gamma \leq \gamma_{\text {conf }}$ and $q>2$, (59, e) is trivially satisfied.

Secondly we claim that (59, a) and (59, c) reduce to the single condition

$$
\frac{\gamma}{q}+\frac{1}{\tilde{q}} \geq \frac{\gamma+1}{2}-\frac{2}{n-1}
$$


in the square

$$
R=\left[\frac{1}{2}-\frac{1}{n-1}, \frac{1}{2}\right) \times\left[\frac{1}{2}-\frac{1}{n-1}, \frac{1}{2}\right) .
$$

More precisely, if $(p, q)$ and $(\tilde{p}, \tilde{q})$ are admissible couples satisfying (59., $\mathrm{c})$, then $\left(\frac{1}{q}, \frac{1}{\tilde{q}}\right)$ is a point in the square $R$ satisfying (60) . Conversely, if $\left(\frac{1}{q}, \frac{1}{\tilde{q}}\right) \in R$ satisfies (60) $)$, then there exists a one parameter family of admissible couples $(p, q)$ and $(\tilde{p}, \tilde{q})$ satisfying (59,c). All these claims can be deduced from the following four quadrant figure.
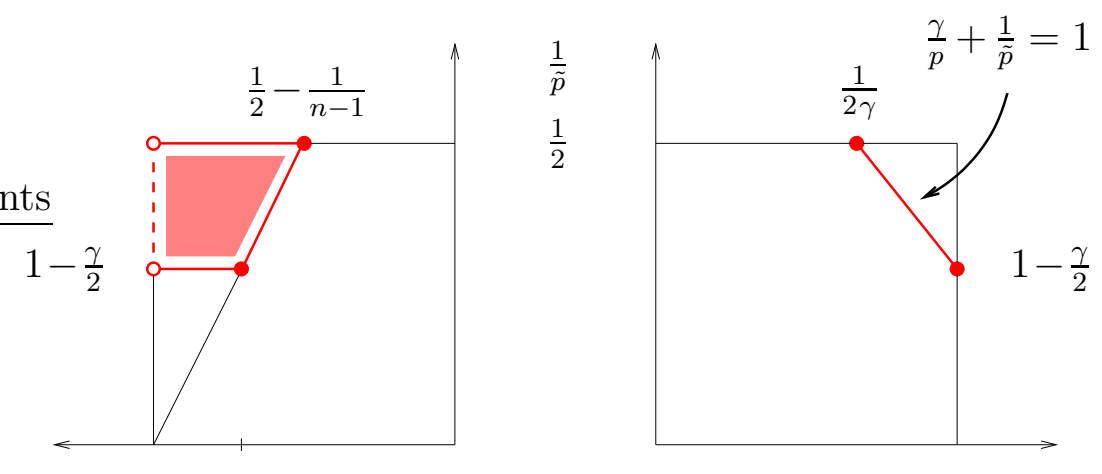

$$
\frac{1}{\tilde{q}} \quad \frac{1}{2} \quad \frac{1}{2}-\frac{2-\gamma}{n-1}
$$
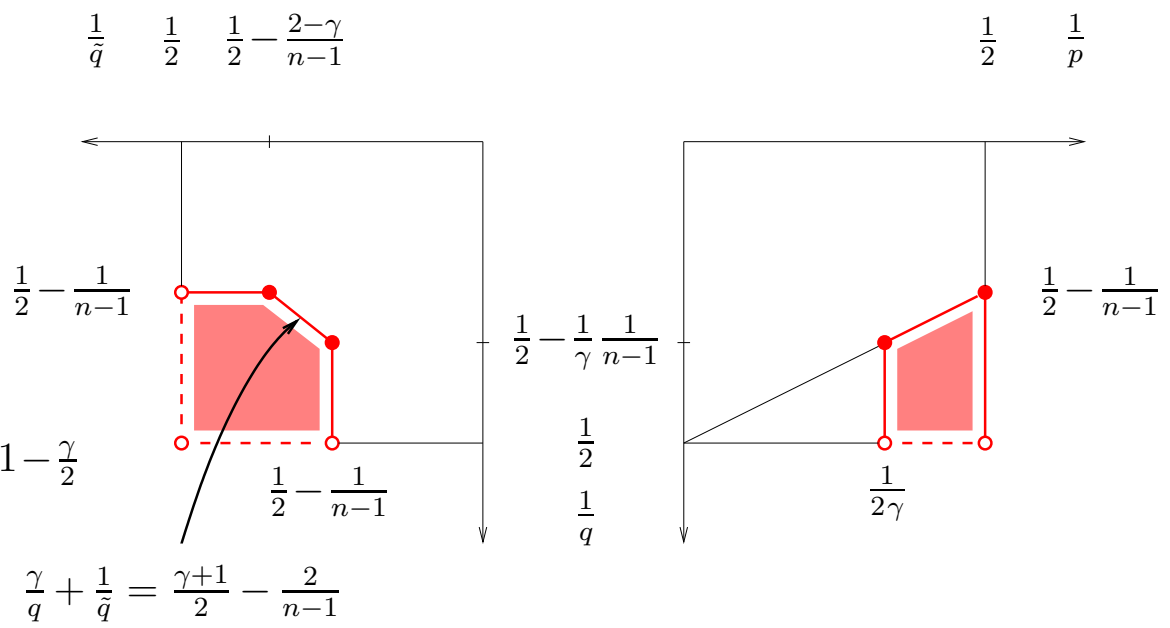

Figure 7. Case $\gamma<2$

Thirdly, as $\gamma \leq \gamma_{\text {conf }}$, (60) follows actually from (59), d.i).

Fourthly we claim that (59,b) follows from (59, d.i) and (59, d.ii). Consider indeed the three lines

$$
\begin{cases}\text { (b) } & \frac{1}{q}+\frac{1}{\tilde{q}}=\frac{n-1}{n+1} \\ \text { (d.i }) & \frac{\gamma}{q}+\frac{1}{\tilde{q}}=1 \\ \text { (d.ii) } & \left(\frac{2 n}{n-1} \gamma-\frac{n+1}{n-1}\right) \frac{1}{q}+\frac{1}{\tilde{q}}=\frac{n+1}{n-1}\end{cases}
$$


in the plane with coordinates $\left(\frac{1}{q}, \frac{1}{\tilde{q}}\right)$. On one hand, they meet at the same point, whose coordinates are

$$
\left\{\begin{array}{l}
\frac{1}{q_{1}}=\frac{2}{n+1} \frac{1}{\gamma-1}, \\
\frac{1}{\tilde{q}_{1}}=\frac{n-1}{n+1}-\frac{2}{n+1} \frac{1}{\gamma-1} .
\end{array}\right.
$$

On the other hand, the coefficients of $\frac{1}{q}$ occur in increasing order in (62) :

$$
1<\gamma<\frac{2 n}{n-1} \gamma-\frac{n+1}{n-1}
$$

Hence (59, b) follows from (59, d.i) and (59, d.ii), which define the sector $S$ with vertex $\left(\frac{1}{q_{1}}, \frac{1}{\tilde{q}_{1}}\right)$ and edges (62, d.i), (62, d.ii) depicted in Figure 8.

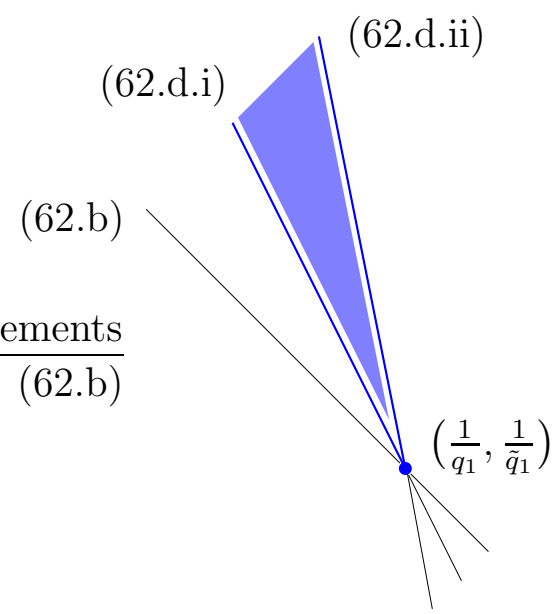

FiguRE 8. Sector $S$

In summary, the set of conditions (59) reduce to the three conditions (59.,d.i), (59.,d.ii), (61) in the plane with coordinates $\left(\frac{1}{q}, \frac{1}{\tilde{q}}\right)$. In order to conclude, we examine the possible intersections of the sector $S$ defined by (59, d.i) and (59), d.ii) with the square $R$ defined by (61), and we determine in each case the minimal regularity $\sigma=\frac{n+1}{2}\left(\frac{1}{2}-\frac{1}{q}\right)$. 
- Case 1: $1<\gamma \leq \gamma_{1}$

In the following three subcases, the minimal regulatity condition is $\sigma>0$, as $\frac{1}{q}>\frac{1}{2}$ can be chosen arbitrarily close to $\frac{1}{2}$.

○ Subcase 1.1: $1<\gamma \leq 1+\frac{2}{n}$

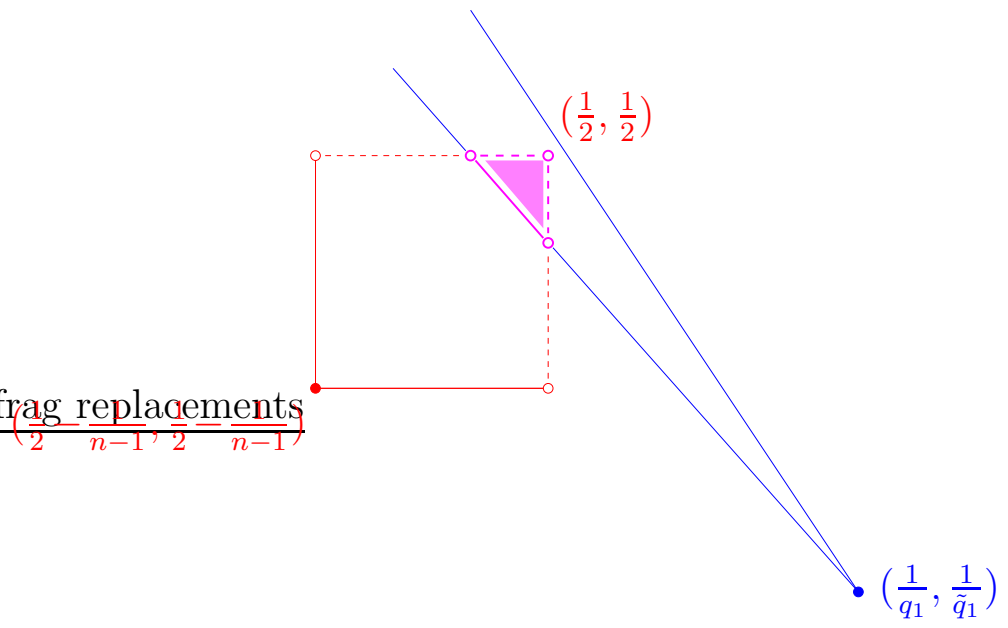

Figure 9. Case $1<\gamma \leq 1+\frac{2}{n}$

○ Subcase 1.2: $1+\frac{2}{n} \leq \gamma \leq 1+\frac{2}{n-1}$

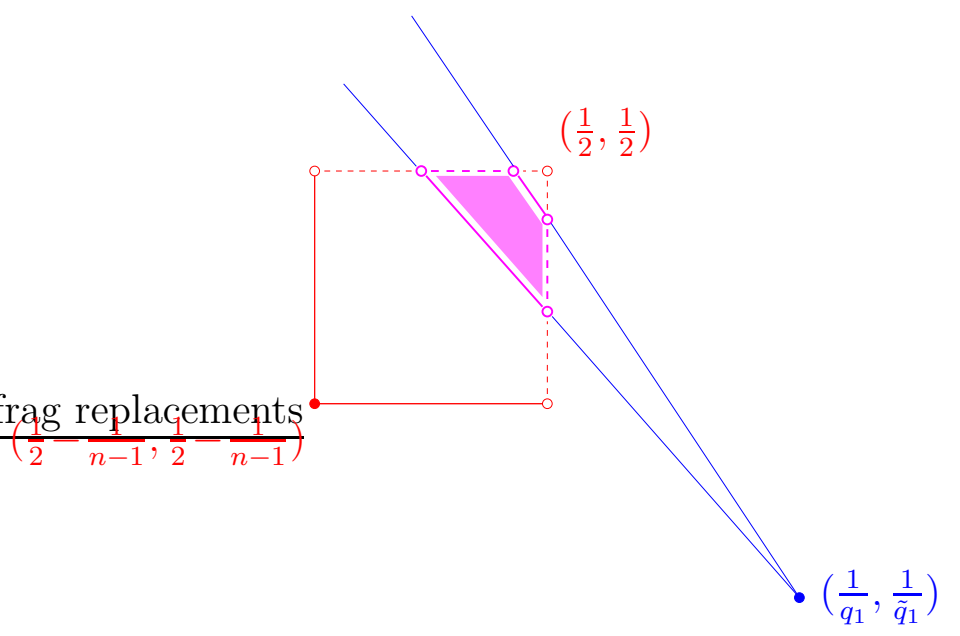

Figure 10. Case $1+\frac{2}{n} \leq \gamma \leq 1+\frac{2}{n-1}$ 
- Subcase 1.3: $1+\frac{2}{n-1} \leq \gamma \leq \gamma_{1}$

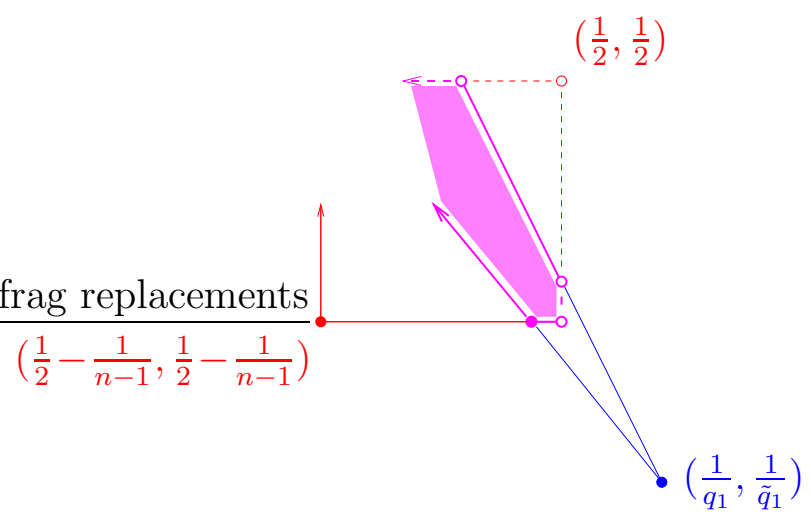

Figure 11. Case $1+\frac{2}{n-1} \leq \gamma \leq \gamma_{1}$

- Case 2: $\gamma_{1}<\gamma \leq \gamma_{2}$

The minimal regularity $\sigma=\sigma_{1}(\gamma)$ is reached at the boundary point $\left(\frac{1}{q}, \frac{1}{\tilde{q}}\right)=\left(\frac{n+5}{4 n} \frac{1}{\gamma-\frac{n+1}{2 n}}\right.$, $\left.\frac{1}{2}-\frac{1}{n-1}\right)$.

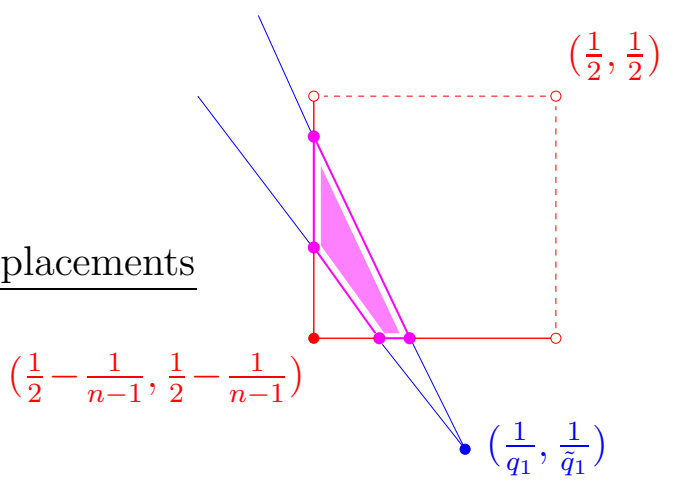

Figure 12. Case $\gamma_{1}<\gamma \leq \gamma_{2}$

- Case 3: $\gamma_{2} \leq \gamma \leq \gamma_{\text {conf }}$

The minimal regularity $\sigma=\sigma_{2}(\gamma)$ is reached at the vertex $\left(\frac{1}{q_{1}}, \frac{1}{\tilde{q}_{1}}\right)$.

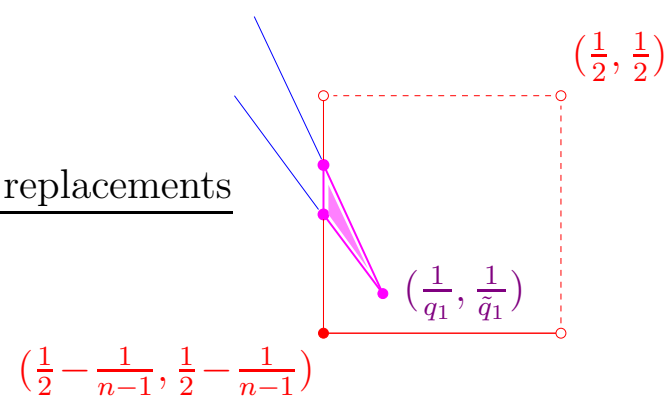

Figure 13. Case $\gamma_{2} \leq \gamma \leq \gamma_{\text {conf }}$ 
In the limit case $\gamma=\gamma_{\text {conf }}$, notice that all indices $\frac{1}{q_{1}}, \frac{1}{\tilde{q}_{1}}, \frac{1}{p_{1}}=\frac{n-1}{2}\left(\frac{1}{2}-\frac{1}{q_{1}}\right), \frac{1}{\tilde{p}_{1}}=\frac{n-1}{2}\left(\frac{1}{2}-\frac{1}{\tilde{q}_{1}}\right)$ become equal to the Strichartz index $\frac{1}{2} \frac{n-1}{n+1}=\frac{1}{2}-\frac{1}{n+1}$.

This concludes the proof of Theorem 6.1 for $1<\gamma \leq \gamma_{\text {conf }}$ and $n \geq 6$.

- Assume that $n=4$ or 5 .

Let us adapt the proof above. If $\gamma \geq 2$, (59.,e) must be checked and (59, a), (59, c) reduce again to (60), but this time in the slightly larger square

$$
R=\left[\frac{1}{2}-\frac{1}{n-1}, \frac{1}{2}\right) \times\left[\frac{1}{2}-\frac{1}{n-1}, \frac{1}{2}\right] .
$$

See Figure 14,
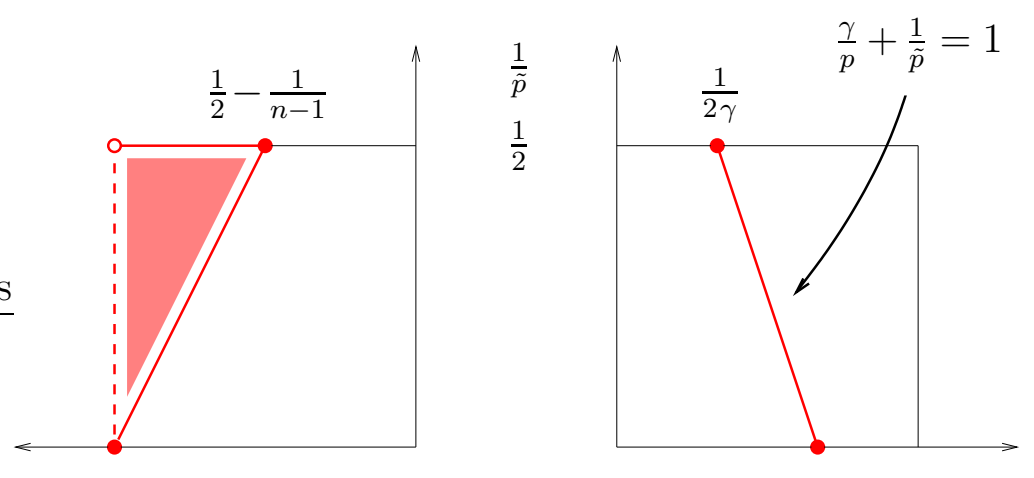

$$
\frac{1}{\tilde{q}} \quad \frac{1}{2}
$$
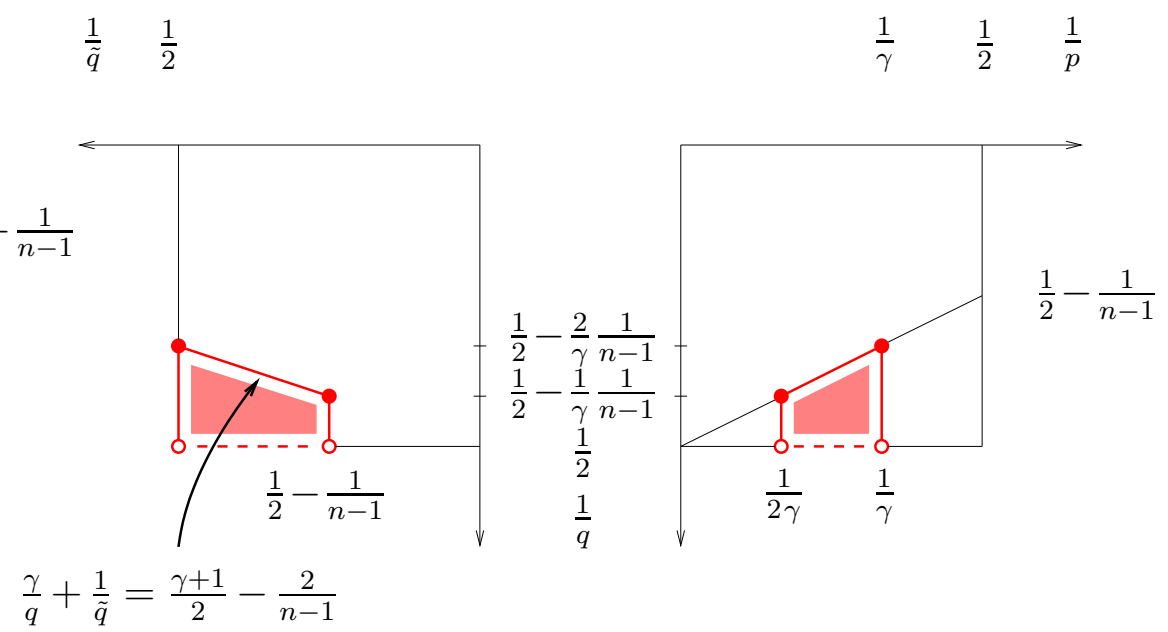

Figure 14. Case $\gamma \geq 2$

Thus (59) reduce to

$$
\begin{cases}\text { (59, d.i), (59, d.ii), (64) } & \text { if } 1<\gamma<2, \\ \text { (59, d.i), (59, d.ii), (59,e), (64) } & \text { if } 2 \leq \gamma \leq \gamma_{\text {conf }}\end{cases}
$$

The case-by-case study of the intersection $S \cap R$ is carried out as above and yield the same results. The only difference lies in the fact that the sector $S$ exists the square $R$ 
through the top edge instead of the left edge (see Figures 15, 16, 17 below). Notice that (59).e) is satisfied, as $q_{1}>\gamma$ when $2 \leq \gamma \leq \gamma_{\text {conf }}$.

- Case 2: $\gamma_{1}<\gamma \leq \gamma_{2}$

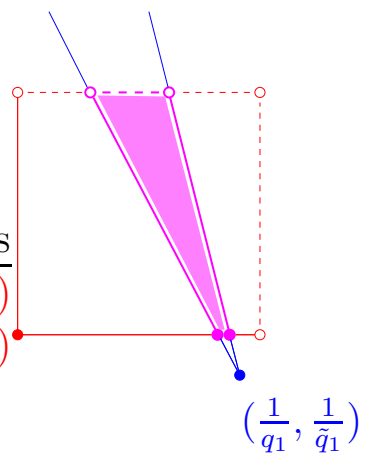

Figure 15. Case $\gamma_{1}<\gamma \leq \gamma_{2}$

- Case 3: $\gamma_{2} \leq \gamma<\gamma_{\text {conf }}$

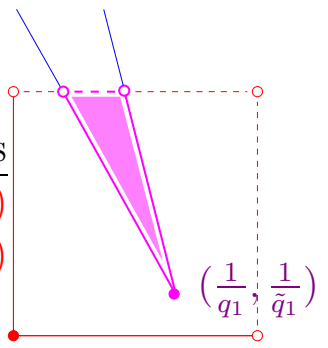

FiguRE 16. Subcase $\gamma_{2} \leq \gamma<2$

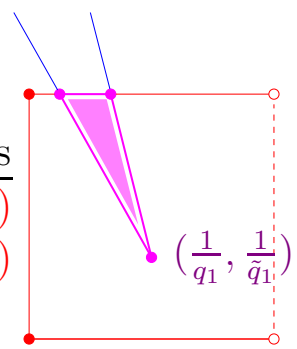

Figure 17. Subcase $2 \leq \gamma \leq \gamma_{\text {conf }}$

This concludes the proof of Theorem 6.1 for $1<\gamma \leq \gamma_{\text {conf }}$ and $n=4,5$.

- Assume that $n=3$.

The proof works the same, except that the square becomes

$$
R= \begin{cases}\left(0, \frac{1}{2}\right) \times\left(0, \frac{1}{2}\right) & \text { if } 1<\gamma<2, \\ \left(0, \frac{1}{2}\right) \times\left(0, \frac{1}{2}\right] & \text { if } 2 \leq \gamma \leq \gamma_{\text {conf }}\end{cases}
$$


and that $\left(\frac{1}{q_{1}}, \frac{1}{\tilde{q}_{1}}\right)$ enters the square $R$ through the vertex $\left(\frac{1}{2}, 0\right)$ instead of the bottom edge. This happens when $\gamma=2$ and in this case (59.e) is satisfied.

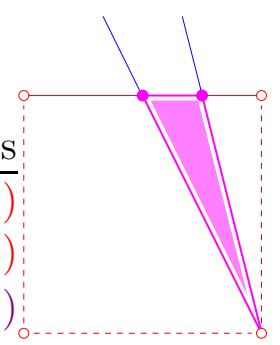

Figure 18. Case $\gamma=2$

It is further satisfied when $2<\gamma \leq \gamma_{\text {conf }}$, as $q_{1}>\gamma$.

This concludes the proof of Theorem 6.1 for $1<\gamma \leq \gamma_{\text {conf }}$.

Proof of Theorem 6.1 for $\gamma_{\text {conf }} \leq \gamma \leq \gamma_{4}$. We resume the fixed point method above, using Corollary 5.3 instead of Theorem [5.2, and obtain in this way the set of conditions:

$$
\left\{\begin{array}{l}
\text { (a) } 2 \leq p \leq \infty \text { and } 2 \leq q<\infty \text { satisfy } \frac{1}{p} \leq \frac{n-1}{2}\left(\frac{1}{2}-\frac{1}{q}\right) \\
\text { (ã) } 2 \leq \tilde{p} \leq \infty \text { and } 2 \leq \tilde{q}<\infty \text { satisfy } \frac{1}{\tilde{p}} \leq \frac{n-1}{2}\left(\frac{1}{2}-\frac{1}{\tilde{q}}\right) \\
\text { (b) } \sigma \geq n\left(\frac{1}{2}-\frac{1}{q}\right)-\frac{1}{p}, \tilde{\sigma} \geq n\left(\frac{1}{2}-\frac{1}{\tilde{q}}\right)-\frac{1}{\tilde{p}}, \sigma+\tilde{\sigma} \leq 1 \\
\text { (c) } \frac{\gamma}{p}+\frac{1}{\tilde{p}}=1 \\
\text { (d) } 1 \leq \frac{\gamma}{q}+\frac{1}{\tilde{q}} \leq 1+\frac{1-\sigma-\tilde{\sigma}}{n} \\
\text { (e) } q>\gamma
\end{array}\right.
$$

We may assume that

$$
\sigma=n\left(\frac{1}{2}-\frac{1}{q}\right)-\frac{1}{p} \quad \text { and } \quad \tilde{\sigma}=n\left(\frac{1}{2}-\frac{1}{\tilde{q}}\right)-\frac{1}{\tilde{p}} .
$$

With this choice, the conditions

$$
\sigma+\tilde{\sigma} \leq 1 \quad \text { and } \quad \frac{\gamma}{q}+\frac{1}{\tilde{q}} \leq 1+\frac{1-\sigma-\tilde{\sigma}}{n}
$$

become

$$
\frac{1}{p}+\frac{1}{\tilde{p}}+1 \geq n\left(1-\frac{1}{q}-\frac{1}{\tilde{q}}\right)
$$

and

$$
\frac{1}{p}+\frac{1}{\tilde{p}}+1 \geq(\gamma-1) \frac{n}{q} .
$$

Notice moreover that (67) follows from (68), combined with $\frac{\gamma}{q}+\frac{1}{\tilde{q}} \geq 1$, and that (68) can be rewritten as follows, using (66. c) :

$$
\frac{1}{p}+\frac{n}{q} \leq \frac{2}{\gamma-1} .
$$


Thus (66) reduces to the set of conditions

$$
\left\{\begin{array}{l}
\text { (a) } 2 \leq p \leq \infty \text { and } 2 \leq q<\infty \text { satisfy } \frac{1}{p} \leq \frac{n-1}{2}\left(\frac{1}{2}-\frac{1}{q}\right) \\
\text { (ã) } 2 \leq \tilde{p} \leq \infty \text { and } 2 \leq \tilde{q}<\infty \text { satisfy } \frac{1}{\tilde{p}} \leq \frac{n-1}{2}\left(\frac{1}{2}-\frac{1}{\tilde{q}}\right) \\
\text { (c) } \frac{\gamma}{p}+\frac{1}{\tilde{p}}=1 ; \\
\text { (d.i) } \frac{\gamma}{q}+\frac{1}{\tilde{q}} \geq 1 ; \\
\text { (d.ii) } \frac{1}{p}+\frac{n}{q} \leq \frac{2}{\gamma-1} \\
\text { (e) } q>\gamma
\end{array}\right.
$$

Among these conditions, consider first (69, a) and (69, d.ii). In the plane with coordinates $\left(\frac{1}{p}, \frac{1}{q}\right)$, the two lines

$$
\left\{\begin{array}{l}
\text { (a) } \frac{1}{p}+\frac{n-1}{2} \frac{1}{q}=\frac{n-1}{4} \\
\text { (d.ii) } \frac{1}{p}+\frac{n}{q}=\frac{2}{\gamma-1}
\end{array}\right.
$$

meet at the point $\left(\frac{1}{p_{2}}, \frac{1}{q_{2}}\right)$ given by

$$
\left\{\begin{array}{l}
\frac{1}{p_{2}}=\frac{n-1}{n+1}\left(\frac{n}{2}-\frac{2}{\gamma-1}\right), \\
\frac{1}{q_{2}}=\frac{1}{n+1}\left(\frac{4}{\gamma-1}-\frac{n-1}{2}\right) .
\end{array}\right.
$$

As $\gamma$ varies between $\gamma_{\text {conf }}$ and $\gamma_{3}$, this point moves on the line (70.a) between the Strichartz point $\left(\frac{1}{2}-\frac{1}{n+1}, \frac{1}{2}-\frac{1}{n+1}\right)$ and the Keel-Tao endpoint $\left(\frac{1}{2}, \frac{1}{2}-\frac{1}{n-1}\right)$, where it exists the square $\left[0, \frac{1}{2}\right] \times\left(0, \frac{1}{2}\right]$. Thus $[69$,a) and [69, d.ii) determine the following regions:

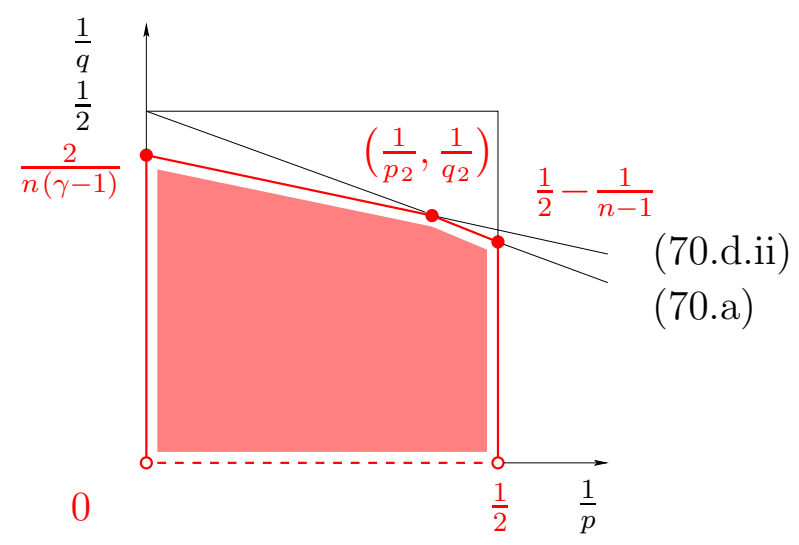

Figure 19. Case 4: $\gamma_{\text {conf }} \leq \gamma \leq \gamma_{3}$ 


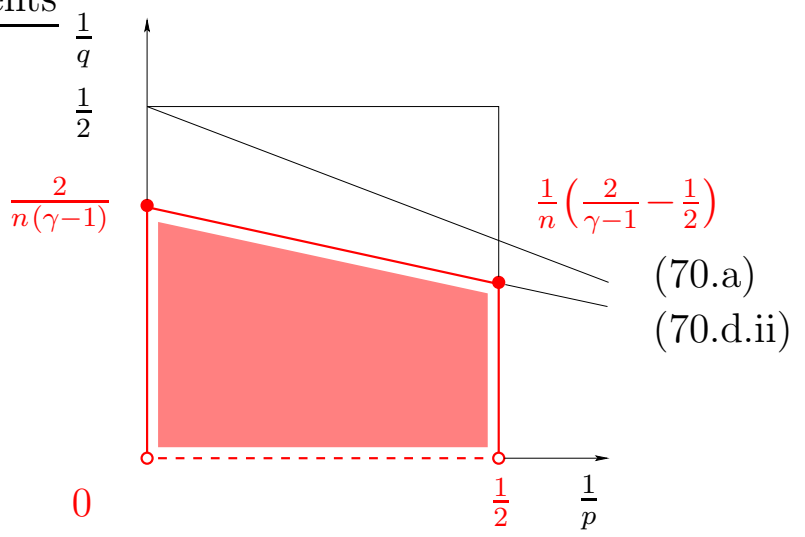

Figure 20. Case 5: $\gamma_{3} \leq \gamma \leq \gamma_{4}$

For later use, notice that the minimal regularity

$$
\sigma=\frac{n}{2}\left(\frac{1}{2}-\frac{1}{q}\right)-\frac{1}{p} \geq \sigma_{3}(\gamma)
$$

is reached on the boundary line $(70$, d.ii) and that

$$
p_{2}<2 \gamma \text {. }
$$

This inequality holds indeed when $\gamma=\gamma_{\text {conf }}$ and it remains true as $\gamma$ increases while $p_{2}$ decreases.

Let us next discuss all conditions (69), first in high dimensions and next in low dimensions.

- Assume that $n \geq 6$.

Firstly notice that (69.e) is trivially satisfied in this case. On one hand, we have indeed $\gamma \leq \gamma_{4} \leq 2$. On the other hand, it follows from (69), d.ii) that

Hence $\gamma \leq 2<q$.

$$
\frac{1}{q} \leq \frac{2}{n(\gamma-1)} \leq \frac{2}{n\left(\gamma_{\mathrm{conf}}-1\right)}=\frac{1}{2}\left(1-\frac{1}{n}\right)<\frac{1}{2} \text {. }
$$

Secondly we claim that (69.)a), (69, ã), (69, c), (69, d.ii) reduce to the conditions

$$
\left\{\begin{array}{l}
\text { (a) } \frac{\gamma}{q}+\frac{1}{\tilde{q}} \leq \frac{\gamma+1}{2}-\frac{2}{n-1} \\
\text { (d.ii) } \frac{\gamma}{q}+\frac{n-1}{2 n} \frac{1}{q} \leq \frac{n+3}{4 n}+\frac{2}{n} \frac{1}{\gamma-1}
\end{array}\right.
$$

in the rectangle

$$
R=\left(0, \frac{1}{n}\left(\frac{2}{\gamma-1}-\frac{1}{2 \gamma}\right)\right] \times\left(0, \frac{1}{2}-\frac{2-\gamma}{n-1}\right] .
$$

Actually they even reduce to the single condition (74, d.ii) if $\gamma \geq \gamma_{3}$. All these claims are obtained again by examining the following four quadrant figures. 


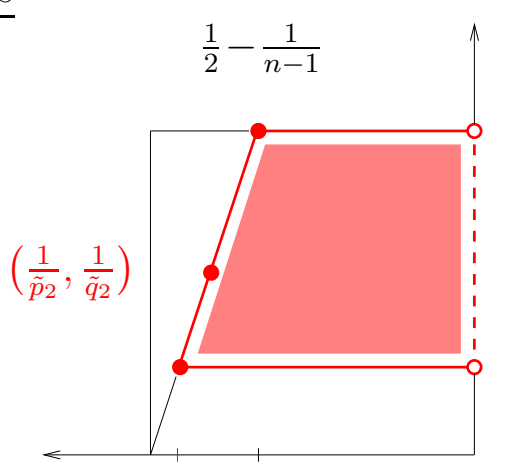

$\frac{1}{\tilde{q}} \quad \frac{1}{2} \quad \frac{1}{2}-\frac{2-\gamma}{n-1}$

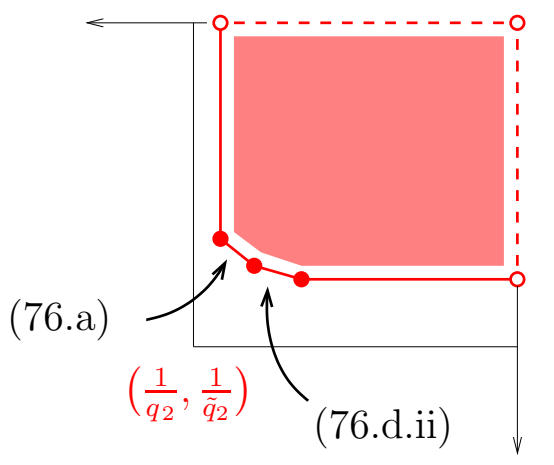

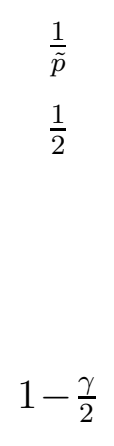

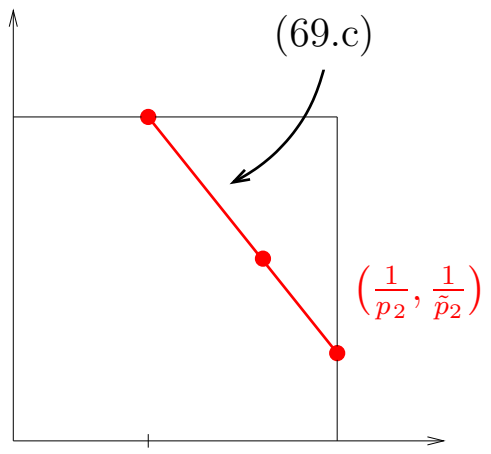

$\frac{1}{2 \gamma} \quad \frac{1}{2} \quad \frac{1}{p}$

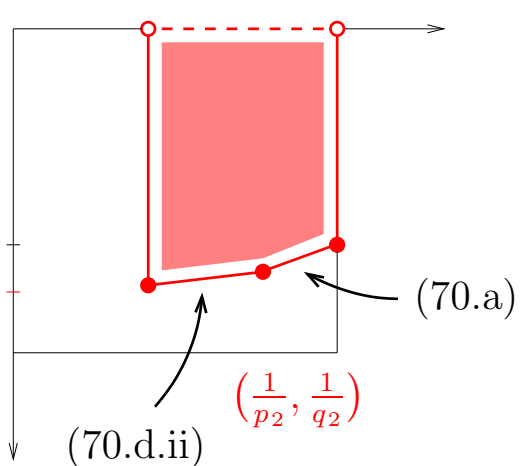

Figure 21. Case 4: $\gamma_{\text {conf }} \leq \gamma \leq \gamma_{3}$ 

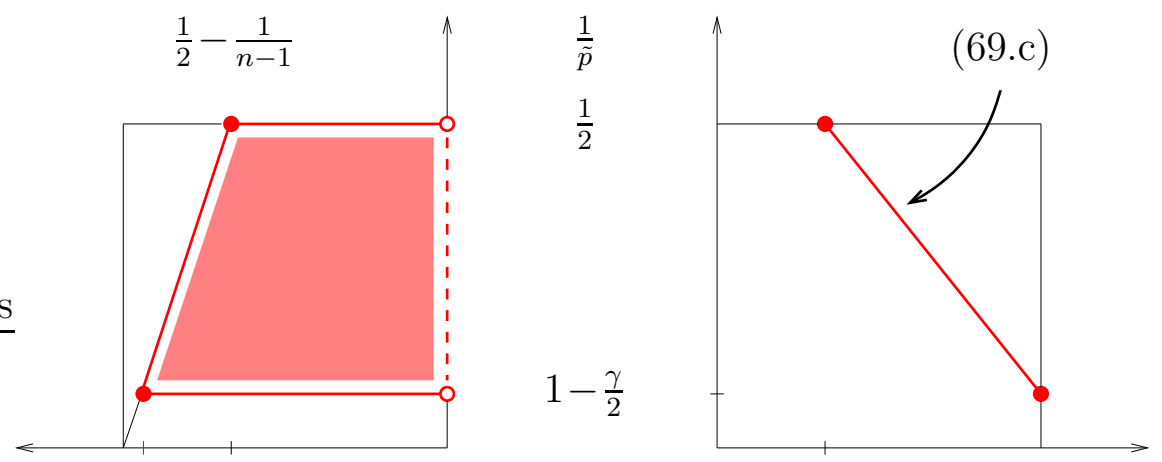

$\frac{1}{\tilde{q}} \quad \frac{1}{2} \quad \frac{1}{2}-\frac{2-\gamma}{n-1}$
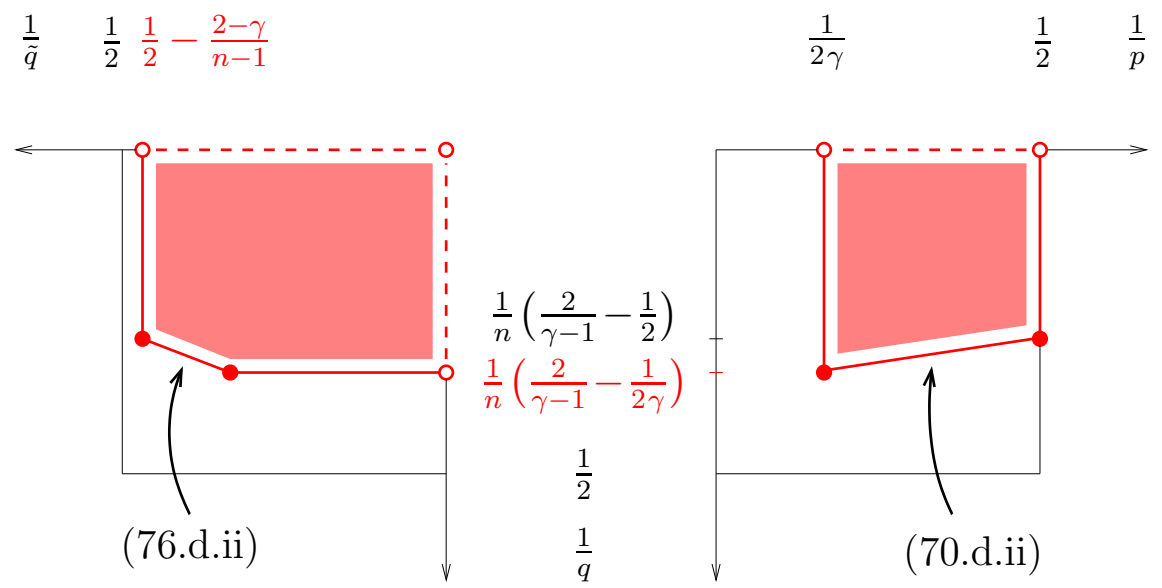

Figure 22. Case 5: $\gamma_{3} \leq \gamma \leq \gamma_{4}$ 
Thirdly, in the plane with coordinates $\left(\frac{1}{q}, \frac{1}{\tilde{q}}\right)$, the conditions (69, d.i), (74,a), (74, d.ii) define the convex region $C$ in Figure 23 with edges

$$
\left\{\begin{array}{l}
\text { (a) } \frac{\gamma}{q}+\frac{1}{\tilde{q}}=\frac{\gamma+1}{2}-\frac{2}{n-1} \\
\text { (d.i) } \frac{\gamma}{q}+\frac{1}{\tilde{q}}=1 \\
\text { (d.ii) } \frac{\gamma}{q}+\frac{n-1}{2 n} \frac{1}{\tilde{q}}=\frac{n+3}{4 n}+\frac{2}{n} \frac{1}{\gamma-1},
\end{array}\right.
$$

and with vertices given by

$$
\begin{cases}\frac{1}{q_{2}}=\frac{4}{n+1} \frac{1}{\gamma-1}-\frac{1}{2} \frac{n-1}{n+1}, & \frac{1}{\tilde{q}_{2}}=\frac{n}{n+1} \gamma-\frac{4}{n+1} \frac{1}{\gamma-1}+\frac{1}{2}-\frac{2}{n-1}-\frac{4}{n+1}, \\ \frac{1}{q_{3}}=\frac{4}{n+1} \frac{1}{\gamma-1}-\frac{1}{2} \frac{n+3}{n+1} \frac{1}{\gamma}, & \frac{1}{\tilde{q}_{3}}=\frac{3}{2} \frac{n-1}{n+1}-\frac{4}{n+1} \frac{1}{\gamma-1} .\end{cases}
$$

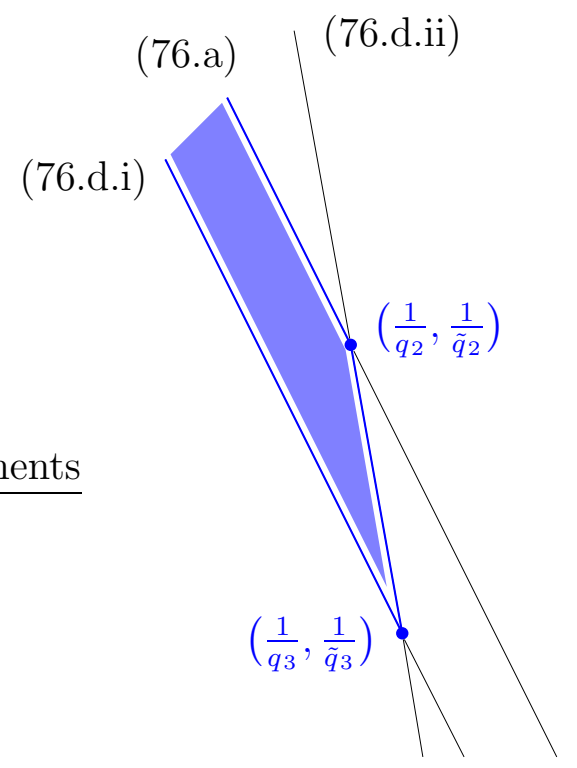

Figure 23. Convex region $C$

In order to conclude, it remains for us to determine the possible intersections of the convex region $C$ above with the rectangle $R$ defined by (75) and in each case the minimal regularity $\sigma=n\left(\frac{1}{2}-\frac{1}{q}\right)-\frac{1}{p}$. 
- Case 4: $\gamma_{\text {conf }} \leq \gamma \leq \gamma_{3}$

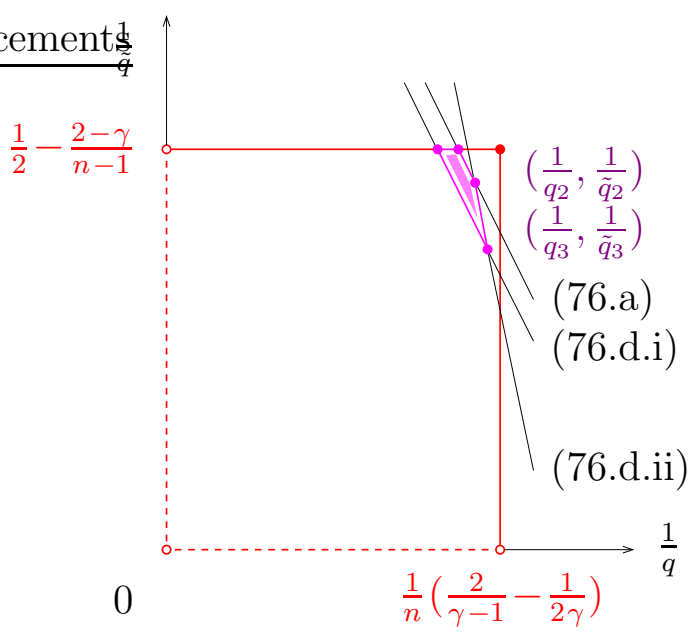

Figure 24. Case 4: $\gamma_{\text {conf }} \leq \gamma \leq \gamma_{3}$

- Case 5: $\gamma_{3} \leq \gamma \leq \gamma_{4}$

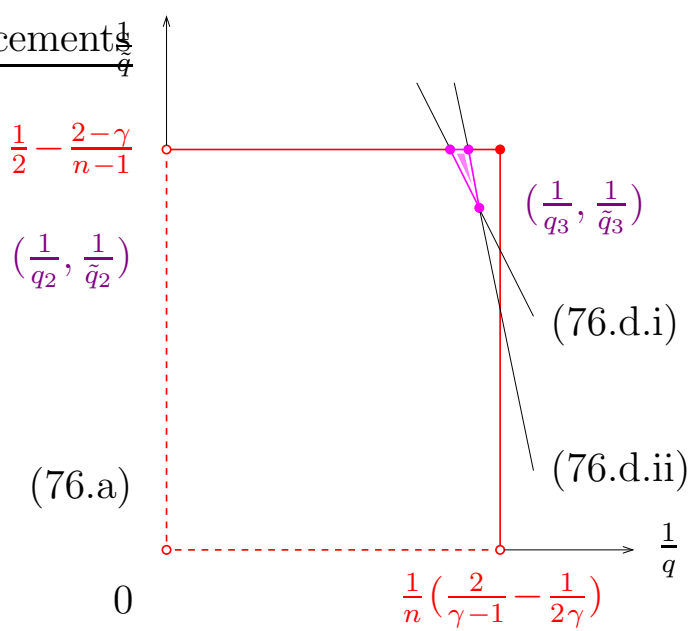

Figure 25. Case 5: $\gamma_{3} \leq \gamma \leq \gamma_{4}$

In both cases, the minimal regularity $\sigma=\sigma_{3}(\gamma)$ is reached when $\left(\frac{1}{p}, \frac{1}{q}\right)$ and $\left(\frac{1}{q}, \frac{1}{\tilde{q}}\right)$ lie on the edges (70,d.ii) and (76, d.ii). See Figures 21 and 22, This concludes the proof of Theorem 6.1 for $\gamma_{\text {conf }}<\gamma \leq \gamma_{4}$ and $n \geq 6$.

- Assume that $3 \leq n \leq 5$.

Then $\gamma \geq \gamma_{\text {conf }} \geq 2$ and the four quadrant figures become 


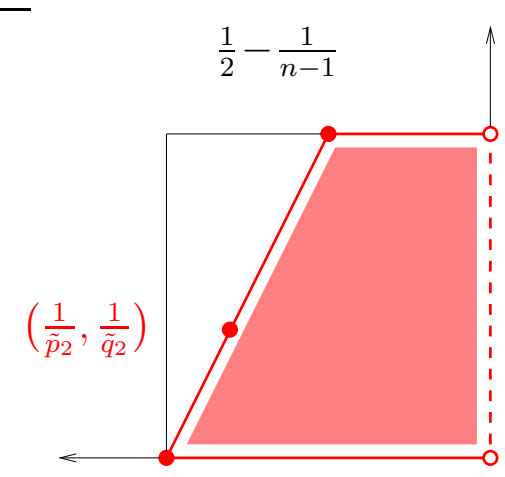

$\frac{1}{\tilde{q}} \quad \frac{1}{2}$

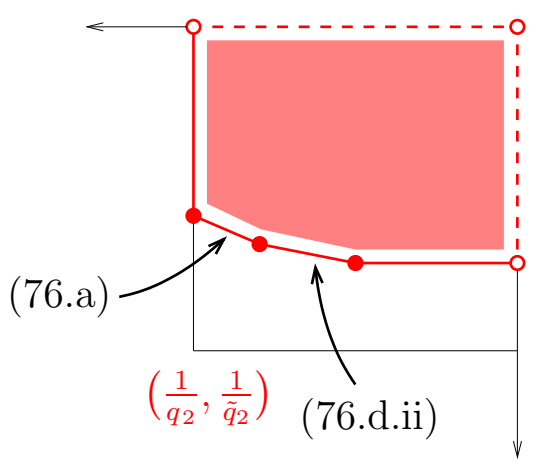

$$
\begin{aligned}
& \frac{1}{\tilde{p}} \\
& \frac{1}{2}
\end{aligned}
$$

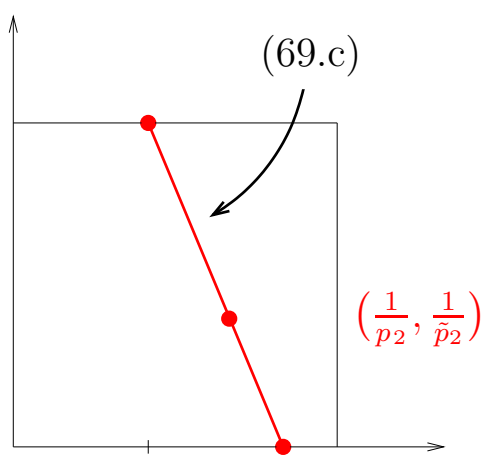

$\frac{1}{2 \gamma} \quad \frac{1}{\gamma} \quad \frac{1}{2} \quad \frac{1}{p}$

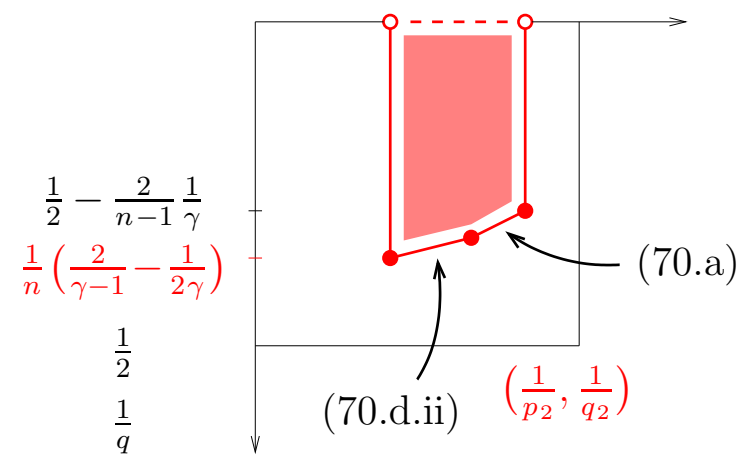

Figure 26. Case 4 : $\gamma_{\text {conf }} \leq \gamma \leq \gamma_{3}$ 


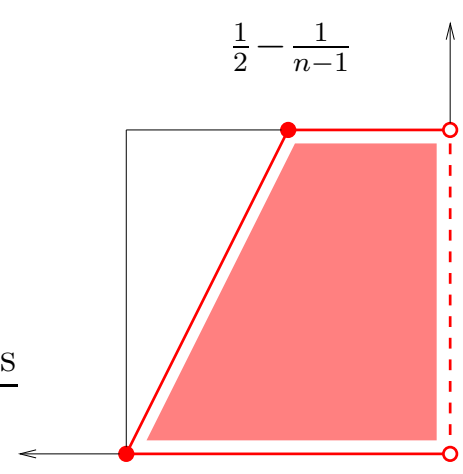

$\frac{1}{\tilde{q}} \quad \frac{1}{2}$

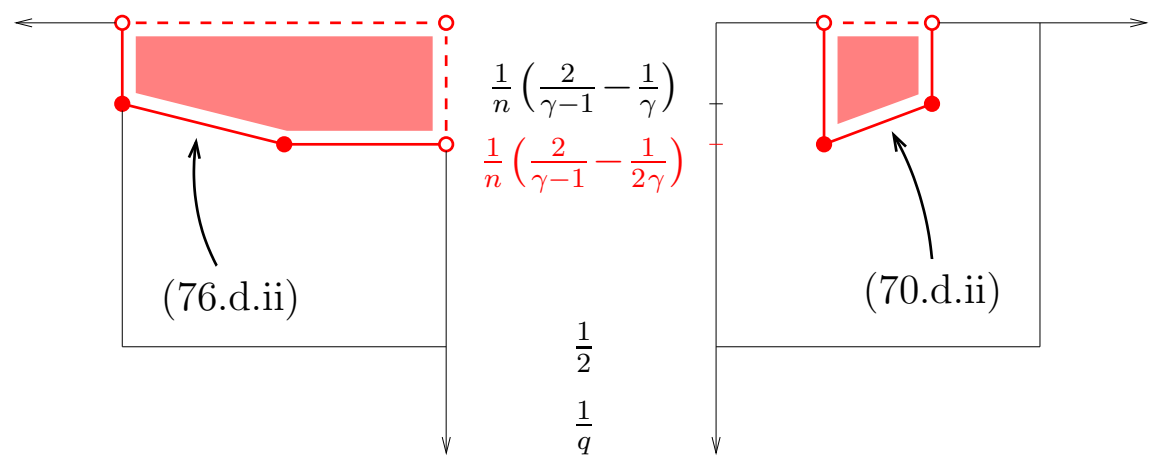

Figure 27. Case 5: $\gamma_{3} \leq \gamma \leq \gamma_{4}$ 
Consequently the four conditions (69, a), (69, ã), (69, c), (69, d.ii) reduce again to the two conditions (74. a), (74. d.ii) if $\gamma_{\text {conf }} \leq \gamma \leq \gamma_{3}$, and actually to the single condition (74.).ii) if $\gamma_{3} \leq \gamma \leq \gamma_{4}$, but this time in the rectangle

$$
R=\left(0, \frac{1}{n}\left(\frac{2}{\gamma-1}-\frac{1}{2 \gamma}\right)\right] \times\left(0, \frac{1}{2}\right] .
$$

Moreover (69).e) is satisfied, as $\frac{1}{q} \leq \frac{1}{n}\left(\frac{2}{\gamma-1}-\frac{1}{2 \gamma}\right)<\frac{1}{\gamma}$.

We conclude again by examining the possible intersections $C \cap R$ of the convex region defined by (69), d.i), (74,a), (74, d.ii) with the rectangle (78) and by determining in each case the minimal regularity $\sigma=n\left(\frac{1}{2}-\frac{1}{q}\right)-\frac{1}{p}$.

- Case 4: $\gamma_{\mathrm{conf}} \leq \gamma \leq \gamma_{3}$

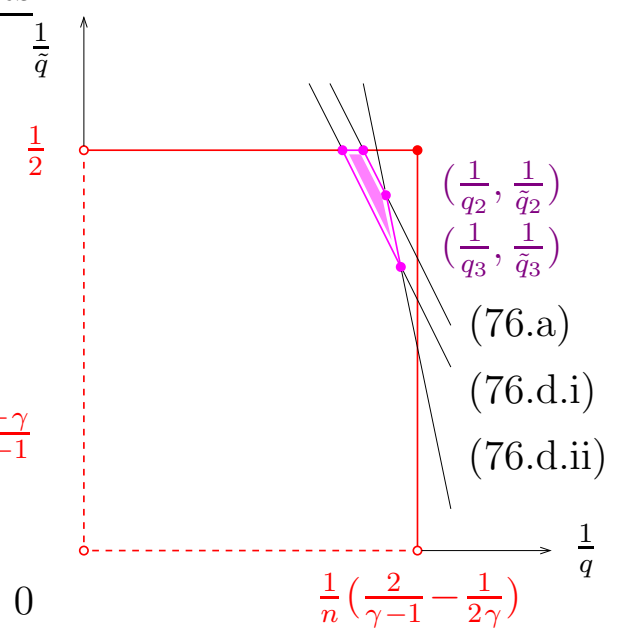

Figure 28. Case 4: $\gamma_{\text {conf }} \leq \gamma \leq \gamma_{3}$

- Case 5: $\gamma_{3} \leq \gamma \leq \gamma_{4}$

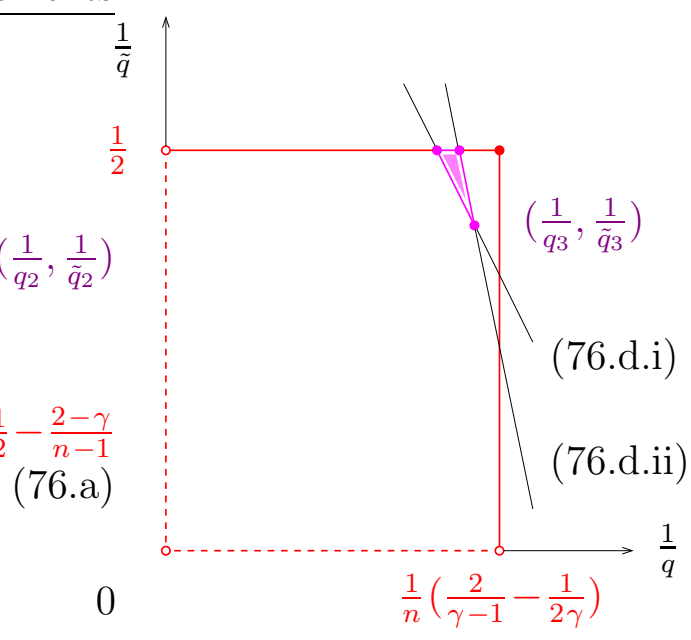

Figure 29. Case 5: $\gamma_{3} \leq \gamma \leq \gamma_{4}$ 
In both cases, the minimal regularity $\sigma=\sigma_{3}(\gamma)$ is reached again when $\left(\frac{1}{p}, \frac{1}{q}\right)$ and $\left(\frac{1}{q}, \frac{1}{\tilde{q}}\right)$ lie on the edges (70,d.ii) and (76, d.ii). See Figures 26 and 27. This concludes the proof of Theorem 6.1 for $\gamma_{\text {conf }}<\gamma \leq \gamma_{4}$ and $3 \leq n \leq 5$.

Remark 6.3. In dimension $n=3$, Metcalfe and Taylor [27] obtain a global existence result beyond $\gamma=\gamma_{4}$. In a forthcoming work, we shall deal with this case in higher dimensions.

Remark 6.4. In dimension $n=2$, the statement of Theorem (6.1) holds true with (53) replaced by

$$
\begin{cases}\sigma=0^{+} & \text {if } 1<\gamma \leq 2, \\ \sigma=\tilde{\sigma}_{1}(\gamma)^{+} & \text {if } 2 \leq \gamma \leq 3, \\ \sigma=\sigma_{2}(\gamma) & \text { if } 3<\gamma<5 \\ \sigma=\sigma_{3}(\gamma)^{+} & \text {if } 5 \leq \gamma<\infty\end{cases}
$$

where $\tilde{\sigma}_{1}(\gamma)=\frac{3}{4}-\frac{3}{2} \frac{1}{\gamma}$. Notice that the condition $q>\gamma$ is not redundant if $2<\gamma<3$ and that it is actually responsible for the curve $\tilde{C}_{1}$.

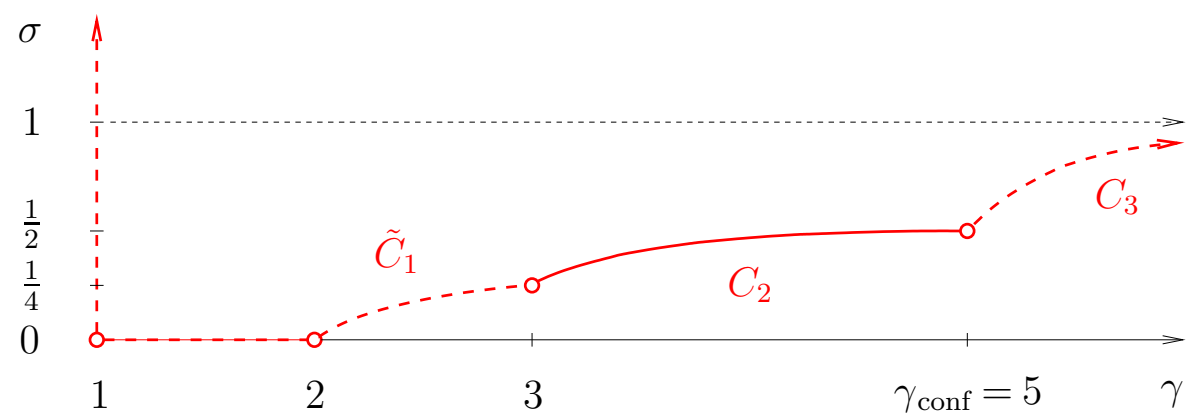

FiguRE 30. Regularity for GWP on $\mathbb{H}^{2}$

\section{Appendix A}

In this appendix we collect some lemmata in Fourier analysis on $\mathbb{R}$, which are used in the kernel analysis carried out in Section 3.

Lemma A.1. Consider the oscillatory integral

$$
I(t, x)=\int_{-\infty}^{+\infty} d \lambda a(\lambda) e^{i t \phi(\lambda)}
$$

where the phase is given by

$$
\phi(\lambda)=\sqrt{\lambda^{2}+\kappa^{2}}-\frac{x \lambda}{t}
$$

(recall that $\kappa$ is a fixed constant $>0$ ) and the amplitude $a \in \mathcal{C}_{c}^{\infty}(\mathbb{R})$ has the following behavior at the origin

$$
a(\lambda)=O\left(\lambda^{2}\right)
$$


Then

$$
|I(t, x)| \lesssim \frac{1+|x|}{(1+|t|)^{3 / 2}} \quad \forall|x| \leq \frac{|t|}{2} .
$$

Proof. Let us compute the first two derivatives

$$
\phi^{\prime}(\lambda)=\frac{\lambda}{\sqrt{\lambda^{2}+\kappa^{2}}}-\frac{x}{t} \quad \text { and } \quad \phi^{\prime \prime}(\lambda)=\kappa^{2}\left(\lambda^{2}+\kappa^{2}\right)^{-\frac{3}{2}} .
$$

The phase $\phi$ has a single stationary point:

$$
\lambda_{0}=\kappa \frac{x}{t}\left(1-\frac{x^{2}}{t^{2}}\right)^{-\frac{1}{2}},
$$

which remains bounded under our assumption $|x| \leq \frac{|t|}{2}$ :

$$
\left|\lambda_{0}\right| \leq \frac{\kappa}{\sqrt{3}} \leq \kappa
$$

For later use, let us compute

$$
\phi\left(\lambda_{0}\right)=\kappa\left(1-\frac{x^{2}}{t^{2}}\right)^{\frac{1}{2}} \quad \text { and } \quad \phi^{\prime \prime}\left(\lambda_{0}\right)=\kappa^{-1}\left(1-\frac{x^{2}}{t^{2}}\right)^{\frac{3}{2}} .
$$

Since $\phi^{\prime \prime}>0$, we can perform a global change of variables $\lambda \longleftrightarrow \mu$ on $\mathbb{R}$ so that

$$
\phi(\lambda)-\phi\left(\lambda_{0}\right)=\mu^{2}
$$

Specifically,

$$
\mu=\epsilon(\lambda)\left(\lambda-\lambda_{0}\right)
$$

where

$$
\epsilon(\lambda)=\left\{\int_{0}^{1} d s(1-s) \phi^{\prime \prime}\left((1-s) \lambda_{0}+s \lambda\right)\right\}^{\frac{1}{2}} .
$$

This way, our oscillatory integral becomes

$$
I(t, x)=e^{i t \phi\left(\lambda_{0}\right)} \int_{\mathbb{R}} d \mu \tilde{a}(\mu) e^{(-1+i t) \mu^{2}},
$$

where

$$
\tilde{a}(\mu)=\frac{d \lambda}{d \mu} a(\lambda(\mu)) e^{\mu^{2}}
$$

is again a smooth function with compact support, whose derivatives are controlled uniformly in $t$ and $x$, as long as $|x| \leq \frac{|t|}{2}$. Using Taylor's formula, let us expand

$$
\tilde{a}(\mu)=\sum_{j=0}^{3} \tilde{a}_{j} \mu^{j}+\tilde{a}_{4}(\mu) \mu^{4},
$$

where

$$
\tilde{a}_{0}=\left(\frac{2}{\phi^{\prime \prime}\left(\lambda_{0}\right)}\right)^{\frac{1}{2}} a\left(\lambda_{0}\right)=\mathrm{O}\left(\lambda_{0}^{2}\right)=\mathrm{O}\left(\frac{x^{2}}{t^{2}}\right),
$$

the other constants $\tilde{a}_{1}, \tilde{a}_{2}, \tilde{a}_{3}$ and the function $\tilde{a}_{4}(\mu)$, as well as its derivatives, are bounded uniformly in $t$ and $x$. Let us split up accordingly

$$
I(t, x)=\sum_{j=0}^{4} I_{j}(t, x)
$$

where

$$
I_{j}(t, x)=\tilde{a}_{j} e^{i t \phi\left(\lambda_{0}\right)} \int_{\mathbb{R}} d \mu \mu^{j} e^{(-1+i t) \mu^{2}} \quad(j=0,1,2,3)
$$


and

$$
I_{4}(t, x)=e^{i t \phi\left(\lambda_{0}\right)} \int_{\mathbb{R}} d \mu \tilde{a}_{4}(\mu) \mu^{4} e^{(-1+i t) \mu^{2}} .
$$

The first and third expressions are handled by elementary complex integration:

$$
\begin{aligned}
& I_{0}(t, x)=\tilde{a}_{0} \sqrt{\pi} e^{i t \phi\left(\lambda_{0}\right)}(1-i t)^{-\frac{1}{2}}=\mathrm{O}\left(\frac{x^{2}}{t^{2}}(1+|t|)^{-\frac{1}{2}}\right)=\mathrm{O}\left(\frac{1+|x|}{(1+|t|)^{3 / 2}}\right), \\
& I_{2}(t, x)=\tilde{a}_{2} \frac{\sqrt{\pi}}{2} e^{i t \phi\left(\lambda_{0}\right)}(1-i t)^{-\frac{3}{2}}=\mathrm{O}\left((1+|t|)^{-3 / 2}\right) .
\end{aligned}
$$

The expressions $I_{1}(t, x)$ and $I_{3}(t, x)$ vanish by oddness. The expression $I_{4}(t, x)$ is obviously bounded by the finite integral

$$
\int_{\mathbb{R}} d \mu \mu^{4} e^{-\mu^{2}}
$$

In order to improve this estimate when $|t|$ is large, let us split up

$$
\int_{\mathbb{R}} d \mu=\int_{|\mu| \leq|t|^{-1 / 2}} d \mu+\int_{|\mu|>|t|^{-1 / 2}} d \mu
$$

The first integral is easily estimated, using the uniform boundedness of $\tilde{a}_{4}(\mu)$ :

$$
\left|\int_{|\mu| \leq|t|^{-1 / 2}} d \mu \tilde{a}_{4}(\mu) \mu^{4} e^{(-1+i t) \mu^{2}}\right| \lesssim \int_{|\mu| \leq|t|^{-1 / 2}} d \mu \mu^{4} \lesssim|t|^{-\frac{5}{2}}
$$

After two integration by parts, using $\mu e^{(-1+i t) \mu^{2}}=-\frac{1}{2(1-i t)} \frac{\partial}{\partial \mu} e^{(-1+i t) \mu^{2}}$, the second integral is estimated by

$$
|t|^{-\frac{5}{2}}+|t|^{-2} \int_{\mathbb{R}} d \mu(1+|\mu|)^{2} e^{-\mu^{2}}
$$

Altogether

$$
I_{4}(t, x)=\mathrm{O}\left((1+|t|)^{-2}\right)
$$

and this concludes the proof of Lemma A.1.

Lemma A.2. Consider the oscillatory integral

$$
J(t, x)=\int_{-\infty}^{+\infty} d \lambda a(\lambda) e^{i t \phi(\lambda)}
$$

where the phase is given again by

$$
\phi(\lambda)=\sqrt{\lambda^{2}+\kappa^{2}}-\frac{x \lambda}{t}
$$

and the amplitude $a(\lambda)$ is now a symbol (of any order) on $\mathbb{R}$, which vanishes on the interval $[-\kappa, \kappa]$. Then

$$
J(t, x)=\mathrm{O}\left(|t|^{-\infty}\right) \quad \forall 0 \leq|x| \leq \frac{|t|}{2} .
$$

Proof. According to (81), (821) and (83),

- $\phi$ has a single stationary point $\lambda_{0} \in\left[-\frac{\kappa}{\sqrt{3}}, \frac{\kappa}{\sqrt{3}}\right]$, which remains away from the support of $a$,

- $\left|\phi^{\prime}(\lambda)\right|=\left|\frac{\lambda}{\sqrt{\lambda^{2}+\kappa^{2}}}-\frac{x}{t}\right| \geq \frac{1}{\sqrt{2}}-\frac{1}{2}>0$ on $\operatorname{supp} a$, 
- $\phi^{\prime \prime}$ is a symbol of order -3 .

These facts allow us to perform several integrations by parts based on

$$
e^{i t \phi(\lambda)}=\frac{1}{i t \phi^{\prime}(\lambda)} \frac{\partial}{\partial \lambda} e^{i t \phi(\lambda)}
$$

and to reach the conclusion.

\section{APPENDix B}

In this appendix, we recall the definition of Sobolev spaces on $\mathbb{H}^{n}$ and some related inequalities. We refer to [33] for more details about function spaces on Riemannian manifolds.

Let $\sigma \in \mathbb{R}$ and $1<q<\infty$. Then $H^{\sigma, q}\left(\mathbb{H}^{n}\right)$ denotes the image of $L^{q}\left(\mathbb{H}^{n}\right)$ under $(-\Delta)^{-\frac{\sigma}{2}}$ (in the space of distributions on $\mathbb{H}^{n}$ ), equipped with the norm

$$
\|f\|_{H^{\sigma, q}}=\left\|(-\Delta)^{-\frac{\sigma}{2}} f\right\|_{L^{q}} .
$$

In this definition, we may replace $(-\Delta)^{-\frac{\sigma}{2}}$ by $D^{-\sigma}=\left(-\Delta-\rho^{2}+\kappa^{2}\right)^{-\frac{\sigma}{2}}$, as long as $\kappa>2\left|\frac{1}{2}-\frac{1}{q}\right| \rho$, in particular by $\tilde{D}^{-\sigma}=\left(-\Delta-\rho^{2}+\tilde{\kappa}^{2}\right)^{-\frac{\sigma}{2}}$, since $\tilde{\kappa}>\rho$. If $\sigma=N$ is a nonnegative integer, then $H^{\sigma, q}\left(\mathbb{H}^{n}\right)$ coïncides with the Sobolev space

$$
W^{N, q}\left(\mathbb{H}^{n}\right)=\left\{f \in L^{q}\left(\mathbb{H}^{n}\right) \mid \nabla^{j} f \in L^{q}\left(\mathbb{H}^{n}\right) \forall 1 \leq j \leq N\right\}
$$

defined in terms of covariant derivatives. In the $L^{2}$ setting, we write $H^{\sigma}\left(\mathbb{H}^{n}\right)$ instead of $H^{\sigma, 2}\left(\mathbb{H}^{n}\right)$.

Proposition B.1 (Sobolev embedding theorem). Let $1<q_{1}, q_{2}<\infty$ and $\sigma_{1}, \sigma_{2} \in \mathbb{R}$ such that $\sigma_{1}-\sigma_{2} \geq \frac{n}{q_{1}}-\frac{n}{q_{2}} \geq 0$. Then

$$
H^{\sigma_{1}, q_{1}}\left(\mathbb{H}^{n}\right) \subset H^{\sigma_{2}, q_{2}}\left(\mathbb{H}^{n}\right) .
$$

By this inclusion, we mean that there exists a constant $C \geq 0$ such that

$$
\|f\|_{H^{\sigma_{2}, q_{2}}} \leq C\|f\|_{H^{\sigma_{1}, q_{1}}} \quad \forall f \in C_{c}^{\infty}\left(\mathbb{H}^{n}\right) .
$$

\section{REFERENCES}

[1] J.-Ph. Anker, V. Pierfelice, Nonlinear Schrödinger equation on real hyperbolic spaces, Ann. Inst. H. Poincaré (C) Non Linear Analysis 26 (2009), 1853-1869

[2] J.-Ph. Anker, V. Pierfelice, M. Vallarino, The Schrödinger equation on Damek-Ricci spaces, preprint [hal-00525155, arXiv:1010.2137, Comm. Part. Diff. Eq. (to appear)

[3] J.-Ph. Anker, V. Pierfelice, M. Vallarino, The wave equation on real hyperbolic spaces, preprint [hal-00525251, arXiv:1010.2372]

[4] H. Bahouri, P. Gérard, High frequency approximation of critical nonlinear wave equations, Amer. J. Math. 121 (1999), 131-175

[5] M. Christ, A. Kiselev, Maximal functions associated to filtrations, J. Funct. Anal. 179 (2001), 409-425

[6] P. D'Ancona, V. Georgiev, H. Kubo, Weighted decay estimates for the wave equation, J. Diff. Eq. 177 (2001), 146-208

[7] J. Fontaine, Une équation semi-linéaire des ondes sur $\mathbb{H}^{3}$, C. R. Acad. Sci. Paris Sér. I Mathématiques 319 (1994), 935-948 
[8] J. Fontaine, A semilinear wave equation on hyperbolic spaces, Comm. Partial Diff. Eq. 22 (1997), $633-659$

[9] V. Georgiev, Semilinear hyperbolic equations, Mem. Math. Soc. Japan 7 (2000)

[10] V. Georgiev, H. Lindblad, C. Sogge, Weighted Strichartz estimates and global existence for semilinear wave equations, Amer. J. Math. 119 (1997), 1291-1319

[11] J. Ginibre, G. Velo, Generalized Strichartz inequalities for the wave equation, J. Funct. Anal. 133 (1995), 50-68

[12] J. Ginibre, G. Velo, The global Cauchy problem for the non linear Klein-Gordon equation, Math. Z. 189 (1985), no. 4, 487-505

[13] A. Hassani, Equation des ondes sur les espaces symétriques riemanniens de type non compact, Ph.D. thesis, Université Paris-Ouest / Nanterre (2011).

[14] S. Helgason, Differential geometry, Lie groups, and symmetric spaces, Academic Press (1978), Amer. Math. Soc. (2001)

[15] S. Helgason, Groups and geometric analysis (integral geometry, invariant differential operators, and spherical functions), Academic Press (1984), Amer. Math. Soc. (2002)

[16] S. Helgason, Geometric analysis on symmetric spaces, Amer. Math. Soc. (1994)

[17] L.V. Hörmander, The analysis of linear partial differential operators III (pseudo-differential operators), Springer-Verlag (1985, 1994, 2007)

[18] A.D. Ionescu, Fourier integral operators on noncompact symmetric spaces of real rank one, J. Funct. Anal. 174 (2000), 274-300

[19] A.D. Ionescu, G. Staffilani, Semilinear Schrödinger flows on hyperbolic spaces: Scattering in $H^{1}$, Math. Ann. 345 (2009), 133-158

[20] F. John, Blow-up of solutions of nonlinear wave equations in three space dimensions, Manuscripta Math. 28 (1979), 235-265

[21] L. Kapitanski, Weak and yet weaker solutions of semilinear wave equations, Comm. Partial Diff. Eq. 19 (1994), 1629-1676

[22] M. Keel, T. Tao, Endpoint Strichartz estimates Amer. J. Math. 120 (1998), 955-980

[23] S. Klainerman, G. Ponce, Global, small amplitude solutions to nonlinear evolution equations, Comm. Pure Appl. Math. 36 (1983), 133-141

[24] T.H. Koornwinder, Jacobi functions and analysis on noncompact semisimple Lie groups, in Special functions (group theoretical aspects and applications), R.A. Askey \& al. (eds.), Reidel (1984), $1-85$

[25] H. Lindblad, C. Sogge, On existence and scattering with minimal regularity for semilinear wave equations, J. Funct. Anal. 130 (1995), 357-426

[26] S. Machihara, M. Nakamura, T. Ozawa, Small global solutions for nonlinear Dirac equations, Differential Integral Equations 17 (2004), no. 5-6, 623-636

[27] J. Metcalfe, M.E. Taylor, Nonlinear waves on $3 D$ hyperbolic space, Trans. Amer. Math. Soc. (to appear)

[28] K. Nakanishi, Scattering theory for the nonlinear Klein-Gordon equation with Sobolev critical power, Internat. Math. Res. Notices 1 (1999), 31-60

[29] V. Pierfelice, Weighted Strichartz estimates for the Schrödinger and wave equations on DamekRicci spaces, Math. Z. 260 (2008), 377-392

[30] T. Sideris, Nonexistence of global solutions to semilinear wave equations in high dimensions, J. Diff. Eq. 52 (1984), 378-406

[31] W. Strauss, Nonlinear wave equations, CBMS Regional Conf. Ser. Math. 73, Amer. Math. Soc. (1989)

[32] D. Tataru, Strichartz estimates in the hyperbolic space and global existence for the semilinear wave equation, Trans. Amer. Math. Soc. 353 (2001), 795-807

[33] H. Triebel, Theory of function spaces II, Monographs Math. 84, Birkhäuser (1992) 
Université d'Orléans \& CNRS, Fédération Denis Poisson (FR 2964) \& Laboratoire MAPMO (UMR 6628), Bâtiment de mathématiques - Route de Chartres, B.P. 6759 45067 ORLÉANS CEDEX 2 - FranCE

E-mail address: anker@univ-orleans.fr

Université d'Orléans \& CNRS, Fédération Denis Poisson (FR 2964) \& Laboratoire MAPMO (UMR 6628), BÂtiment de mathématiques - Route de Chartres, B.P. 6759 45067 ORLÉANS CEDEX 2 - FRANCE

E-mail address: vittoria.pierfelice@univ-orleans.fr 